\title{
Arnold diffusion in a driven optical lattice
}

\author{
Yingyue Boretz and L. E. Reichl \\ Center for Complex Quantum Systems and Department of Physics, The University of Texas at Austin, Austin, Texas 78712, USA
}

(Received 9 December 2015; published 11 March 2016)

\begin{abstract}
The effect of time-periodic forces on matter has been a topic of growing interest since the advent of lasers. It is known that dynamical systems with 2.5 or more degrees of freedom are intrinsically unstable. As a consequence, time-periodic driven systems can experience large excursions in energy. We analyze the classical and quantum dynamics of rubidium atoms confined to a time-periodic optical lattice with 2.5 degrees of freedom. When the laser polarizations are orthogonal, the system consists of two 1.5 uncoupled dynamical systems. When laser polarizations are turned away from orthogonal, an Arnold web forms and the dynamics undergoes a fundamental change. For parallel polarizations, we find huge random excursions in the rubidium atom energies and significant entanglement of energies in the quantum dynamics.
\end{abstract}

DOI: 10.1103/PhysRevE.93.032214

\section{INTRODUCTION}

In 1964 Arnold showed that nonintegrable conservative classical systems (even those close to integrability) with more than two degrees of freedom (DoF) are intrinsically unstable [1]. For conservative nonintegrable systems with three or more DoF, the energy surface is covered densely by interconnected resonance lines (an Arnold web) and the system can diffuse throughout the "energy surface" in the high dimensional phase space. Let us consider a system that is near integrability, with a Hamiltonian of the form $H\left(\left\{I_{j}, \phi_{j}\right\}\right)=H_{0}\left(\left\{I_{j}\right\}\right)+$ $\epsilon V\left(\left\{I_{j}, \phi_{j}\right\}\right)$, where $\left\{I_{j}, \phi_{j}\right\},(j=1, \ldots, d)$ are action-angle variables, $H_{0}$ is the Hamiltonian of a nonlinear integrable system, and $\epsilon V$ is a small perturbation that breaks the integrability. Let us assume that the system satisfies the KAM theorem (Kolmogorov [2], Arnold [3], Moser [4]), which applies if the perturbation is smooth and the integrable system is nondegenerate (as is the case for the system considered here). When the perturbation is very small, most of the phase space will consist of nonresonant KAM tori, although slightly deformed from the integrable case. In addition, the phase space will be contain a dense set of resonance lines (the Arnold web) determined by the resonance conditions $\sum_{j} n_{j} \omega_{j}\left(\left\{I_{j}\right\}\right)=0$, where $\omega_{j}=\partial H_{o} / \partial I_{j}$ and $n_{j}$ ranges over all integers. For small $\epsilon$, each resonance line has a width of order (or smaller than) $\sqrt{\epsilon}$, and a stochastic layer associated with it. Trajectories can diffuse along these stochastic layers. Nekhoroshev showed that, for very small $\epsilon$, the diffusion process may take a very long time [5,6]. If $I_{j}(0)$ is the initial value of a diffusing action variable, then in time $\tau_{N}$ it will have diffused a "distance" $\left|I_{j}\left(\tau_{N}\right)-I_{j}(0)\right| \sim \epsilon^{\beta}$ in a time of order $\tau_{N} \sim(1 / \epsilon) \exp \left(1 / \epsilon^{\alpha}\right)$, where $0<\alpha<1$ and $0<\beta<1$. As the strength $\epsilon$ of the perturbation increases, the width of the resonance regions increases until the system reaches the Chirikov regime [7], where resonances not only cross, but also begin to overlap. Then chaotic regions of the phase space, and random diffusion through the phase space, can become global [8-12].

Arnold diffusion has been of particular interest in regard to the stability of the solar system [13], and it has been shown to have a significant effect on the dynamics of a Rydberg atom in the presence of crossed electric and magnetic fields [14]. It has also been associated to instabilities in colliding beam synchrotron particle accelerators [11] and in coupled delta kicked rotors [15]. In periodically driven systems, energy is not conserved, and the effect of Arnold diffusion can be quite dramatic, as we show below.

Arnold diffusion has also been reported to exist in quantum systems. Malyshev and Chizhova [16] have studied the classical and quantum dynamics of two weakly coupled oscillators placed in a time-periodic external field. For the system they considered, which has $2.5 \mathrm{DoF}$, they found diffusion in the quantum system that parallels that in the classical system, but a diffusion rate that was an order of magnitude slower in some parameter regimes. Arnold diffusion has also been reported to increase the conductance of open billiards with three DoF [17]. In classical systems, primary resonances and "daughter" resonances that occur due to interaction between primary resonances form a self-similar network in the phase space, and the diffusion process can occur at all length scales [11]. Quantum systems are primarily influenced by classical structures that occupy a volume in the phase space of order Planck's constant or larger. In resonance regions that are larger than Planck's constant, quantum states can spread throughout resonances and chaotic regions [18,19]. Therefore, it is not surprising that the analog of Arnold diffusion exists in quantum systems, although perhaps on a more subdued scale.

In subsequent sections, we consider the classical and quantum dynamics of the center-of-mass motion of noninteracting two-level atoms (rubidium atoms) confined to a body-centered square optical lattice whose amplitude has a time-periodic modulation. Optical lattices have been realized in the laboratory by several experimental groups [20-23]. The classical static version of the optical lattice considered here was analyzed in Ref. [24] for parameters realizable in experiments involving rubidium atoms [23]. We use those same parameters but introduce a time-periodic modulation (TPM) of the amplitude of the laser beams. As we shall see, the TPM optical lattice has 2.5 DoF and can show Arnold diffusion for a range of its parameters, and the center of mass energy of the atoms need not be conserved. The lattice we consider is also a generalization of the 1.5 DoF TPM optical lattice, with embedded thermal cesium atoms, considered in the Texas experiment [25-27], and the 1.5 DoF optical lattice, with embedded sodium Bose-Einstein condensate, considered in the NIST experiment [27,28]. Those experiments showed the existence of chaos-assisted tunneling in the atomic dynamics of the system. However, in both experiments the TPM optical lattice had only 1.5 DoF and could not show Arnold diffusion. 
The effects of Arnold diffusion may help explain the high harmonic radiation emitted from a carbon nanotube in a numerical study of radiation coming from a nanotube in a monochromatic radiation field [29]. It might also help explain the apparent thermalization and induced phase transition, in a condensed matter system in the presence of laser radiation [30].

We begin in Sec. II with an analysis of the Hamiltonian governing the dynamics of atoms in the optical lattice. We write the Hamiltonian in a form that allows us to follow changes in the dynamics as we change the amplitude of the driving, ranging from zero driving to full driving. In Sec. III we focus on classical dynamics. We write the Hamiltonian in terms of action-angle variables, first for the libration region of the motion and then for the rotation region of the dynamics. This allows us to construct the Arnold web for the two regions and make estimates for the lattice coupling strength at which the dynamics transitions from the Nekhoroshev regime to the Chirikov regime. We then analyze the behavior of the average energy of the particles as a function of lattice coupling strength (angle between electric field polarization of the lattice) and amplitude of the driving. In Sec. IV we analyze the quantum behavior of the lattice. We obtain the Floquet eigenvalues and eigenstates of the system and follow their behavior as a function of the strength of the driving field. We compute the average energy of the Floquet states as a function of driving amplitude and compare that behavior to that of the classical system. Finally, in Sec. V we make some concluding remarks.

\section{THE HAMILTONIAN}

The Hamiltonian, in dimensionless units, for two-level atoms in a two-dimensional TPM optical lattice, can be written (see Appendix A)

$$
\begin{aligned}
H(t)= & p_{x}^{2}+p_{y}^{2}+U \cos ^{2}(\omega t)\left[\cos ^{2}(x)+\cos ^{2}(y)\right. \\
& +b \cos (x) \cos (y)],
\end{aligned}
$$

where $b=2 \hat{\epsilon}_{1} \cdot \hat{\epsilon}_{2}(0 \leqslant b \leqslant 2), U$ is proportional to the intensity of the laser radiation that forms the static optical lattice, and $\hat{\epsilon}_{1}\left(\hat{\epsilon}_{2}\right)$ is the polarization unit vector for the optical lattice along the $x$ direction ( $y$ direction). We want to examine the behavior of the atoms in this lattice, both in the static case and with increasing amplitude of the TPM of the optical lattice amplitude. To this end, we generalize this Hamiltonian and write it in the form

$$
H(t)=p_{x}^{2}+p_{y}^{2}+\left[V_{0}+V_{1} \cos ^{2}(\omega t)\right] V(x, y),
$$

where

$$
V(x, y)=U\left[b \cos (x) \cos (y)+\cos ^{2}(x)+\cos ^{2}(y)\right],
$$

and we require that $V_{0}+V_{1}=1$. The case $V_{1}=0$ corresponds to the 2 DoF static lattice considered in Ref. [24]. For the case $V_{1}=1$, the system corresponds to a $2.5 \mathrm{DoF}$ generalization of the 1.5 DoF lattices considered in Refs. [25-27] and [28]. In subsequent sections, we will always take $U=20$ and $\omega=2 \pi$, although our results can be rescaled as described at the end of Appendix A.

The constant $b$ is a parameter that measures the coupling of the atomic motion in the $x$ and $y$ directions. For the case $b=0$,
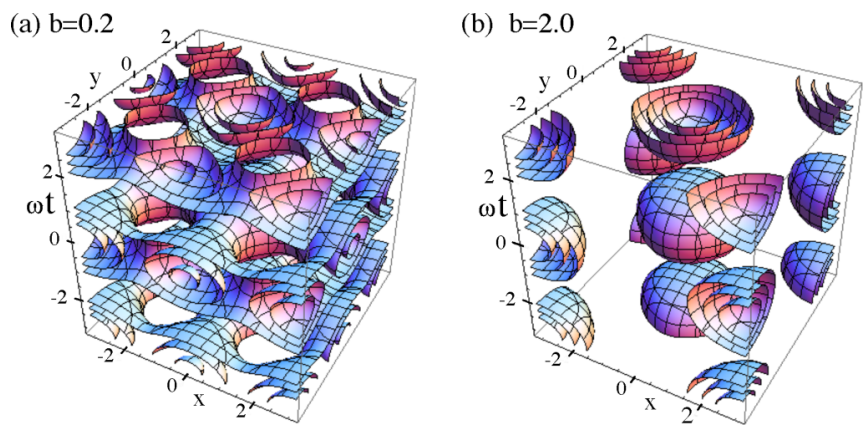

FIG. 1. (a) Contour plot of the potential energy in the unit cell for one period of oscillation of the driving field for (a) $U=20, V_{1}=1.0$, $b=0.2$ and (b) $U=20, V_{1}=1.0, b=2.0$.

the atomic dynamics in the $x$ and $y$ directions is decoupled. For $b=0, V_{1}=0$, the atomic dynamics is integrable and equivalent of two decoupled pendulums. For $b=0$ and $V_{1} \neq 0$, the atomic dynamics in each of the $x$ and $y$ directions are decoupled from one another but can be locally chaotic, and each is equivalent to the $1.5 \mathrm{DoF}$ lattices considered in Refs. [25,27,28]. For the case, $b \neq 0, V_{1} \neq 0$ the dynamics in the $x$ and $y$ directions is coupled, and the potential energy has an amplitude that varies periodically in time, so the atomic motion has 2.5 DoF.

In Fig. 1(a) we show the potential energy in the unit cell of the TPM BCC optical lattice for $b=0.2$ and $V_{1}=1$, as a function of $x, y$, and $\omega t$ (the third coordinate). As was shown in Ref. [24], the static lattice $\left(V_{1}=0\right)$ for $b=0.2$ has potential wells and various saddle points that localize low-energy particles, and some of this structure can be seen in Fig. 1(a), although the amplitude of the potential energy varies periodically in time. In Fig. 1(b) we show the same plot but for $b=2.0$ and $V_{1}=1$. In this case, the potential energy, for a particle traveling along the time axis looks like a soft Lorenz gas that turns on and off. As shown in Ref. [24], for $b=2.0$ and $V_{1}=0$, the lattice is open and dominated by chaos. A similar behavior appears to occur for the case $b=2.0, V_{1}=1$, but with more DoF.

In subsequent sections, we examine first the classical and then quantum behavior of this lattice.

\section{CLASSICAL DYNAMICS}

The first step in analyzing the classical dynamics is to determine if there are fixed points, $\left(\dot{p}_{x}=\dot{p}_{y}=\dot{x}=\dot{y}=0\right)$, in the phase space of the system. Hamilton's equations are given by

$$
\begin{aligned}
\frac{d p_{x}}{d t}= & -\frac{\partial H(t)}{\partial x}=-U\left[V_{1} \cos ^{2}(\omega t)+V_{0}\right][-b \sin (x) \cos (y) \\
& -2 \sin (x) \cos (x)], \\
\frac{d p_{y}}{d t}= & -\frac{\partial H(t)}{\partial y}=-U\left[V_{1} \cos ^{2}(\omega t)+V_{0}\right][-b \cos (x) \sin (y) \\
& -2 \sin (y) \cos (y)], \\
\frac{d x}{d t}= & \frac{\partial H(t)}{\partial p_{x}}=2 p_{x} \quad \text { and } \quad \frac{d y}{d t}=\frac{\partial H(t)}{\partial p_{y}}=2 p_{y} .
\end{aligned}
$$


This time-periodically driven system has the same fixed points as described in Ref. [24] (which corresponds to $V_{1}=0$ ), except that, for $V_{1} \neq 0$, the amplitude of the potential energy varies periodically in time. There are potential energy maxima located at $\left(p_{x}=0, p_{y}=0, x=n_{x} \pi, y=n_{y} \pi\right)$ for $\left(n_{x}=\right.$ $0, \pm 1, \pm 2, \ldots)$ and $\left(n_{y}=0, \pm 1, \pm 2, \ldots\right)$. There are potential energy minima located at $\left(p_{x}=0, p_{y}=0, x=n_{x} \frac{\pi}{2}, y=\right.$ $\left.n_{y} \frac{\pi}{2}\right)$, for $n_{x}$ and $n_{y}$ odd. For $b<2$, there are saddle points located at $\left(p_{x}=0, p_{y}=0, x=n_{x} \pi, y= \pm \cos ^{-1}(b / 2)\right)$ and $\left(p_{x}=0, p_{y}=0, y=n_{y} \pi, x= \pm \cos ^{-1}(b / 2)\right)$. The effect of these various fixed points on the form of the potential energy surface can be seen in Fig. 1(a). For $b=2$, the saddle points disappear and are replaced by fixed lines $y=x \pm \pi$ and $y=-x \pm \pi$, which for the case $V_{1}=0$ open the lattice to unimpeded flow through the potential energy landscape. The open regions of the lattice can be seen in Fig. 1(b).

Because of the nonlinearity of the equations of motion, there are infinite families of resonances between the DoF of the system. The overlap of these resonances leads to chaos in the phase space dynamics in the region of overlap. For the 2 DoF system $\left(b \neq 0, V_{1}=0\right)$, the chaotic regions in different parts of the phase space are isolated by KAM tori and energy is constant. For the two decoupled 1.5 DoF systems $(b=$ $0, V_{1}=1$ ), energy will oscillate or undergo a diffusive random walk, but it does not experience large excursions. For the case $\left(b \neq 0, V_{1} \neq 0\right)$, with $2.5 \mathrm{DoF}$, as pointed out by Arnold [1], KAM tori can no longer isolate regions of the phase space. For small deviations from integrability (small $b$ and $V_{1}$ ), there will be slow diffusion along resonance lines (the Nekhoroshev regime). As $b$ and $V_{1}$ increase, resonances can begin to overlap (the Chirikov regime), and, as we show below, the energy can begin to experience large fluctuations.

\section{A. Hamiltonian in action-angle variables}

It is useful to rewrite the Hamiltonian in the form

$$
\begin{aligned}
H(t)= & p_{x}^{2}+p_{y}^{2}+\frac{U}{2} \cos ^{2}(x)+\frac{U}{2} \cos ^{2}(y) \\
& +b \frac{U}{2} \cos (x) \cos (y)+\frac{U}{2} \cos (2 \omega t) \cos ^{2}(x) \\
& +\frac{U}{2} \cos (2 \omega t) \cos ^{2}(y)+b \frac{U}{2} \cos (2 \omega t) \cos (x) \cos (y)
\end{aligned}
$$

With this form of the Hamiltonian, we can use the transformation to action-angle variables discussed in Aopendix B.
We first give the Hamiltonian for the libration region and then for the rotation region. For simplicity, we do not consider the case where one DoF lies in the libration region and the other in the rotation region. However, the action variables have been defined so that it is possible to follow a transition smoothly into a regime where one DoF is in the libration region and the other is in the rotation region. Since we are not solving equations of motion in terms of action-angle variables, we don't write those two cases here, but it is easily done using the transformations given in Appendix B.

\section{Libration Hamiltonian}

If we perform the canonical transformation $\left(p_{x}, x, p_{y}, y\right) \rightarrow\left(J_{x}, \theta_{x}, J_{y}, \theta_{y}\right)$ discussed in Appendix $\mathrm{B}$, and note that $\cos \left(\frac{\pi}{2}+x\right)=-\sin (x)$ and $\sin \left[\operatorname{am}\left(g_{x}, \kappa_{x}\right)\right]=$ $\operatorname{sn}\left(g_{x}, \kappa_{x}\right)$, the Hamiltonian takes the form

$$
\begin{aligned}
H(t)= & E_{x}+E_{y}+b \frac{U}{2} \kappa_{x} \kappa_{y} \operatorname{sn}\left[f_{x}, \kappa_{x}\right] \operatorname{sn}\left[f_{y}, \kappa_{y}\right] \\
& +\frac{U}{2} \cos (2 \omega t) \kappa_{x}^{2} \operatorname{sn}^{2}\left[f_{x}, \kappa_{x}\right] \\
& +\frac{U}{2} \cos (2 \omega t) \kappa_{y}^{2} \operatorname{sn}^{2}\left[f_{y}, \kappa_{y}\right] \\
& +b \frac{U}{2} \cos (2 \omega t) \kappa_{x} \kappa_{y} \operatorname{sn}\left[f_{x}, \kappa_{x}\right] \operatorname{sn}\left[f_{y}, \kappa_{y}\right],
\end{aligned}
$$

where $\operatorname{sn}\left[f_{x}, \kappa_{x}\right]$ is a Jacobi sn function with modulus $\kappa_{x}, \kappa_{x}^{2}=$ $\frac{2 E_{x}}{U}, \kappa_{y}^{2}=\frac{2 E_{y}}{U}, f_{x}=\frac{2}{\pi} \mathrm{K}\left(\kappa_{x}\right) \theta_{x}$, and $f_{y}=\frac{2}{\pi} \mathrm{K}\left(\kappa_{y}\right) \theta_{y}$, and $\mathrm{K}\left(\kappa_{x}\right)$ is the complete elliptic integral of the first kind. The Jacobi sn function has a series expansion [31]

$$
\operatorname{sn}[z, \kappa]=\sum_{m=0}^{\infty} C_{m}(\kappa) \sin \left[(2 m+1) \frac{\pi z}{2 \mathrm{~K}(\kappa)}\right],
$$

where $C_{m}(\kappa)=\frac{\pi}{\kappa \mathrm{K}(\kappa)} \operatorname{csch}\left[(2 m+1) \frac{\pi}{2} \frac{\mathrm{K}^{\prime}(\kappa)}{\mathrm{K}(\kappa)}\right]$. Some values of $C_{m}(\kappa)$ include $C_{0}(0.999999)=1.2530, C_{0}(0.5)=1.0176$, $C_{0}(0.1)=1.00063, \quad C_{1}(0.999999)=0.3687, \quad C_{1}(0.5)=$ 0.0180, $\quad C_{1}(0.1)=0.0006, \quad C_{2}(0.999999)=0.1546$, $C_{2}(0.5)=0.0003, \quad C_{2}(0.1)=4 \times 10^{-7}$. The values fall off rapidly with increasing $m$.

If we now substitute the series for the Jacobi sn function into the Hamiltonian and combine the trig functions, we can write the Hamiltonian in the form

$$
\begin{aligned}
H(t)= & E_{x}\left(J_{x}\right)+E_{y}\left(J_{y}\right)+\frac{U}{8} \sum_{m_{1}=0}^{\infty} \sum_{m_{2}=0}^{\infty} \sum_{\beta= \pm 1}\left(\kappa_{x}^{2} C_{m_{1}}\left(\kappa_{x}\right) C_{m_{2}}\left(\kappa_{x}\right)\left\{\cos \left[2\left(M_{-} \theta_{x}+\beta \omega t\right)\right]-\cos \left[2\left(M_{+} \theta_{x}+\beta \omega t\right)\right]\right\}\right. \\
& \left.+\kappa_{y}^{2} C_{m_{1}}\left(\kappa_{y}\right) C_{m_{2}}\left(\kappa_{y}\right)\left\{\cos \left[2\left(M_{-} \theta_{y}+\beta \omega t\right)\right]-\cos \left[2\left(M_{+} \theta_{y}+\beta \omega t\right)\right]\right\}\right) \\
& +b \frac{U}{4} \sum_{m_{x}=0 m_{y}=0}^{\infty} \sum_{\beta= \pm 1}^{\infty} \sum_{x} \kappa_{y} C_{m_{x}}\left(\kappa_{x}\right) C_{m_{y}}\left(\kappa_{y}\right)\left\{\left[\beta \cos \left(M_{x} \theta_{x}-\beta M_{y} \theta_{y}\right)\right]+\frac{1}{2} \sum_{\gamma= \pm 1}\left[+\gamma \cos \left(M_{x} \theta_{x}-\gamma M_{y} \theta_{y}+\beta 2 \omega t\right)\right]\right\},
\end{aligned}
$$

where $M_{-}=m_{1}-m_{2}, M_{+}=m_{1}+m_{2}+1, M_{x}=2 m_{x}+1$, and $M_{y}=2 m_{y}+1$.
The Hamiltonain in Eq. (8) contains an infinite number of primary resonances. Furthermore, the interaction between the 
primary resonances gives rise to infinite families of higher order resonances [11]. We can get a rough estimate of the location of the primary resonances. From Hamilton's equations we know that, to zeroth order in $\kappa C_{m}(\kappa)$, we have $\dot{\theta}_{x}=\frac{d E_{x}}{d J_{x}}$ and $\dot{\theta}_{y}=\frac{d E_{y}}{d J_{y}}$. Then the approximate location of the primary resonances is given by
(a) $M_{ \pm} \dot{\theta}_{x}+\beta \omega=0$,
(b) $M_{ \pm} \dot{\theta}_{y}+\beta \omega=0$,
(c) $M_{x} \dot{\theta}_{x}-\beta M_{y} \dot{\theta}_{y}=0$,
(d) $M_{x} \dot{\theta}_{x}-\gamma M_{y} \dot{\theta}_{y}+\beta 2 \omega=0$.

In Fig. 2(a) we have plotted the location of some of these primary resonances for low values of $m_{1}, m_{2}, M_{x}$, and $M_{y}$. This figure shows only a few of the infinite number of primary and higher order resonance lines that form the Arnold web. Note that when $b=0$ all the lines disappear except for the one vertical and one horizontal line. For $b \neq 0$, as the amplitude of the resonance terms grows, either with increasing $U$ or $b$, the region influenced by each resonance widens, and as resonances start to "overlap," chaos appears. This process occurs at all length scales in the classical phase space [11].

\section{Rotation Hamiltonian}

We now perform the canonical transformation $\left(p_{x}, x, p_{y}, y\right) \rightarrow\left(J_{x}, \theta_{x}, J_{y}, \theta_{y}\right)$ for the rotation region as discussed in Appendix B, and note that $\cos \left(\frac{\pi}{2}+x\right)=-\sin (x)$ and $\sin \left[\operatorname{am}\left(g_{x}, \kappa_{x}\right)\right]=\operatorname{sn}\left(g_{x}, \kappa_{x}\right)$, the Hamiltonian takes the form

$$
\begin{aligned}
H(t)= & E_{x}+E_{y}+b \frac{U}{2} \operatorname{sn}\left[g_{x}, \kappa_{x}\right] \operatorname{sn}\left[g_{y}, \kappa_{y}\right] \\
& +\frac{U}{2} \cos (2 \omega t) \operatorname{sn}^{2}\left[g_{x}, \kappa_{x}\right]+\frac{U}{2} \cos (2 \omega t) \operatorname{sn}^{2}\left[g_{y}, \kappa_{y}\right] \\
& +b \frac{U}{2} \cos (2 \omega t) \operatorname{sn}\left[g_{x}, \kappa_{x}\right] \operatorname{sn}\left[g_{y}, \kappa_{y}\right]
\end{aligned}
$$

where $\kappa_{x}^{2}=\frac{U}{2 E_{x}}, \quad \kappa_{y}^{2}=\frac{U}{2 E_{y}}, \quad g_{x}=\frac{2}{\pi} \mathrm{K}\left(\kappa_{x}\right) \theta_{x}, \quad$ and $\quad g_{y}=$ $\frac{2}{\pi} \mathrm{K}\left(\kappa_{y}\right) \theta_{y}$. Using the expansion in Eq. (7), the Hamiltonian can be further reduced to the form

$$
\begin{aligned}
H(t)= & E_{x}\left(J_{x}\right)+E_{y}\left(J_{y}\right)+\frac{U}{8} \sum_{m_{1}=0}^{\infty} \sum_{m_{2}=0}^{\infty} \sum_{\beta= \pm 1}\left(C_{m_{1}}\left(\kappa_{x}\right) C_{m_{2}}\left(\kappa_{x}\right)\left\{\cos \left[2\left(M_{-} \theta_{x}+\beta \omega t\right)\right]-\cos \left[2\left(M_{+} \theta_{x}+\beta \omega t\right)\right]\right\}\right. \\
& \left.+C_{m_{1}}\left(\kappa_{y}\right) C_{m_{2}}\left(\kappa_{y}\right)\left\{\cos \left[2\left(M_{-} \theta_{y}+\beta \omega t\right)\right]-\cos \left[2\left(M_{+} \theta_{y}+\beta \omega t\right)\right]\right\}\right) \\
& +b \frac{U}{4} \sum_{m_{x}=0}^{\infty} \sum_{m_{y}=0}^{\infty} \sum_{\beta= \pm 1} C_{m_{x}}\left(\kappa_{x}\right) C_{m_{y}}\left(\kappa_{y}\right)\left\{\left[\beta \cos \left(M_{x} \theta_{x}-\beta M_{y} \theta_{y}\right)\right]+\frac{1}{2} \sum_{\gamma= \pm 1}\left[+\gamma \cos \left(M_{x} \theta_{x}-\gamma M_{y} \theta_{y}+\beta 2 \omega t\right)\right]\right\}
\end{aligned}
$$

where $M_{-}=m_{1}-m_{2}, M_{+}=m_{1}+m_{2}+1, M_{x}=2 m_{x}+1$, and $M_{y}=2 m_{y}+1$. For the rotation region, the resonance conditions in Eq. (9) also apply, but with different expressions for $\dot{\theta}_{x}$ and $\dot{\theta}_{y}$. The locations of some of the lower order primary resonances for the rotation region are shown in Fig. 2(b). Again, the figure shows only a few of the infinite number of resonance lines that form the Arnold web.

\section{B. Arnold diffusion}

The Hamiltonians in Eqs. (8) and (11) have a structure very similar to the model Hamiltonian studied in Refs. [8,9]. This is easily seen if we replace the time by an angle variable $\phi_{3}=\omega t$ in Eq. (5) and include the corresponding action, $I_{3}$. The Hamiltonian then takes the form

$$
\begin{aligned}
\mathcal{H}= & p_{x}^{2}+p_{y}^{2}+\omega I_{3}+\frac{U}{2} \cos ^{2}(x)+\frac{U}{2} \cos ^{2}(y) \\
& +b \frac{U}{2} \cos (x) \cos (y)+\frac{U}{2} \cos \left(2 \phi_{3}\right) \cos ^{2}(x) \\
& +\frac{U}{2} \cos \left(2 \phi_{3}\right) \cos ^{2}(y)+b \frac{U}{2} \cos \left(2 \phi_{3}\right) \cos (x) \cos (y) .
\end{aligned}
$$

If one writes Hamilton's equations for this system, one finds that $\phi_{3}(t)=\omega t$ and $I_{3}=-H(t) / \omega$, so no new dynamics is involved. This Hamiltonian, when written in terms of action-angle variables [see Eqs. (8) and (11)], contains all harmonics at first order in the parameter $b$, which is the parameter governing the character of the Arnold diffusion.

We can make a very rough estimate of the value of $b$ for which the Nekhoroshev regime transitions to the Chirikov regime. If we plot the primary resonances, $m_{x}=m_{y}=0 \quad\left(M_{x}=M_{y}=1\right)$, for the rotation Hamiltonian in Eq. (11), we can measure the resonance widths $\sqrt{\epsilon}$ and find that they are given by $(b, \sqrt{\epsilon})=(0.002,0.08),(0.02,0.2),(0.2,0.8)$. Therefore, $(b, \epsilon)=(0.002,0.0064),(0.02,0.04),(0.2,0.64)$. The Nekhoroshev estimate of the time for an action variable to change by an amount $\epsilon^{\beta}$ (with $\beta<1$ ) is $\tau_{N}=(1 / \epsilon) \exp \left[1 / \epsilon^{\alpha}\right]$ (with $\alpha<1$ ). As $\epsilon$ decreases, $\tau_{N}$ varies slowly until it reaches a value $\epsilon_{N}$ at which $\tau_{N}$ begins to grow exponentially. When $\alpha=0.5$, the rapid growth in $\tau_{N}$ occurs for $\epsilon_{N} \approx 0.05$, while for $\alpha=0.9$ it occurs for $\epsilon_{N} \approx 0.14$ (we don't have an accurate estimate for $\alpha$ ). Therefore, extrapolating between the measured values of $\epsilon$ for the optical lattice, the transition between the Nekhoroshev and Chirikov regimes (the rapid growth of $\tau_{N}$ ) appears to occur around $0.009 \leqslant b \leqslant 0.02$.

The phenomenon of Arnold diffusion can be seen explicitly in strobe plots of the dynamics if we compare plots for $1.5 \mathrm{DoF}$ systems and 2.5 DoF systems. In Fig. 3(a) we show a strobe plot of $p_{x}$ versus $x$ for the $2 \mathrm{DoF}$ system with Hamiltonian $H_{x}=p_{x}^{2}+\frac{U}{2} \cos ^{2}(x)+\frac{U}{2} \cos (2 \omega t) \cos ^{2}(x)$ and $U=20$. The initial conditions are $x(0)=\frac{\pi}{2}$ and $-30 \leqslant p_{x}(0) \leqslant 30$ with spacing of 0.6. Coordinates $\left(p_{x}, x\right)$ are plotted each period of the oscillation. This Hamiltonian is the same as that 

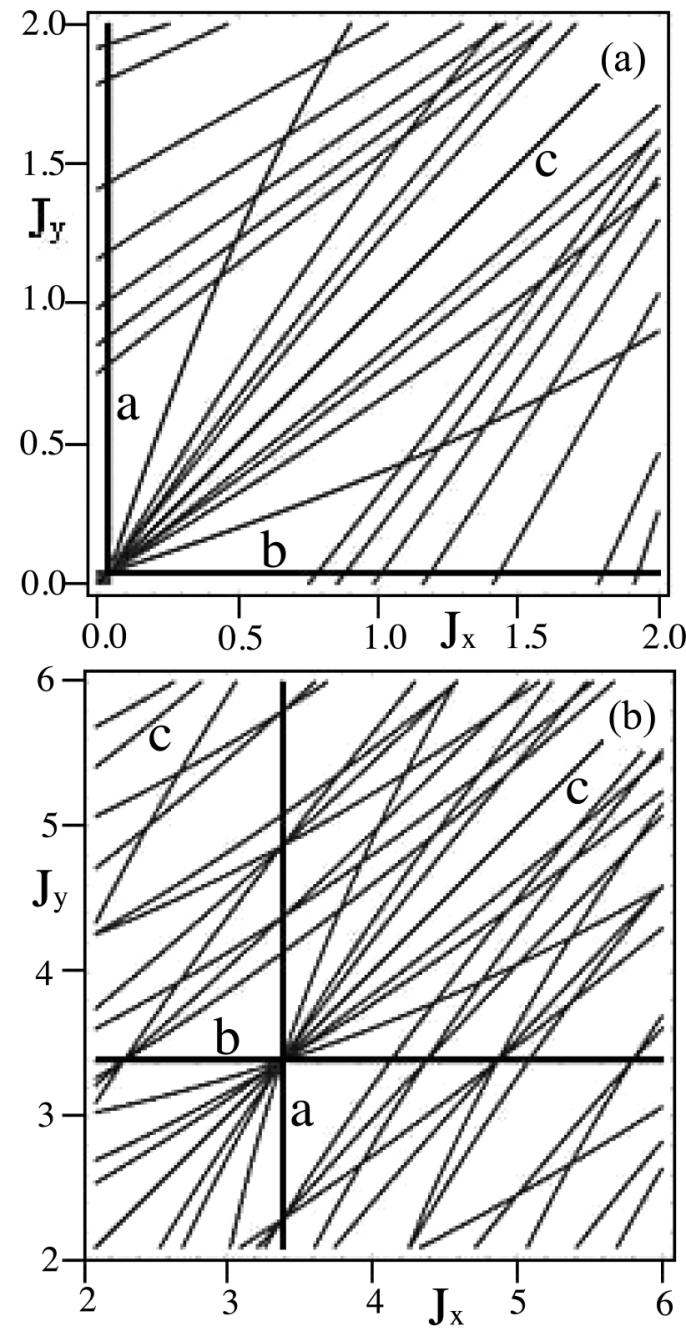

FIG. 2. The Arnold web for $V_{1}=1$. (a) Libration region with $E_{x}<10$ and $E_{y}<10$. The resonances are labeled $a, b$, or $c$ according to Eq. (9). Lines not labeled are type- $d$. All type- $a$ (type- $b$ ) resonances lie on vertical (horizontal) lines. The diagonal line bisecting the figure contains all the type- $c$ resonances. The type- $d$ lines that converge toward the origin correspond to $\pm m_{x} \mp m_{y}= \pm 1$ with $0 \leqslant m_{x}, m_{y} \leqslant 4$. The type- $d$ lines that end along the $J_{x}=0$ or $J_{y}=0$ axes correspond to $m_{x}=m_{y}$ with $0 \leqslant m_{x} \leqslant 7$. (b) Rotation region with $10<E_{x}$ and $10<E_{y}$. The same resonance designations apply except that, in this case, there are additional type- $c$ lines that run parallel to the diagonal line that bisects the figure.

considered in Refs. [25-27]. It has three primary resonances which, for the parameters used here, have overlapped and give rise to the chaotic region shown in the plot. Outside the chaotic region, we see a sequence of KAM tori that block the trajectories from moving to larger positive or negative values of the momentum. In Fig. 3(b) we show a strobe plot with the same initial conditions on $\left(p_{x}, x\right)$, but now with the full Hamiltonian Eq. (5) and initial values $p_{y}=0.5, y(0)=\pi$ and with $b=0$. For $b=0$ there is no coupling between the motions in the $x$ and $y$ DoF and the system evolves as two uncoupled 1.5 DoF systems. Figures 3(a) and 3(b) are essentially indistinguishable. In Figs. 3(c) and 3(d) we show the same strobe plots (same initial conditions) but now with $b=0.002$ and $b=0.02$, respectively, so all DoF are coupled. All four plots in Fig. 3 are run for 2000 periods of oscillation. Weak diffusion across the KAM tori appears to have occurred in Fig. 3(c). However, in Fig. 3(d) diffusion dominates the dynamics. This is consistent with our estimate that the transition between the Nekhoroshev and Chirikov regimes occurs for $0.009 \leqslant b \leqslant 0.02$. In Fig. 3 we see weak diffusion for $b=0.002$ and more rapid diffusion for $b=0.02$.

It is also useful to look at the average energy of the system. In Fig. 3(b) where $b=0$, it is clear that the energy $H(t)$ cannot undergo large excursions. If the initial conditions lie on a KAM torus the energy will undergo small regular oscillations. If they lie in the chaotic region, the energy $H(t)$ can undergo small apparently random oscillations, but they are blocked by KAM tori from large excursions in energy. However, if $b \neq 0$, then, from Figs. 3(c) and 3(d) it is clear that the energy $H(t)$ might undergo large energy oscillations because the KAM tori no longer isolate regions of the phase space from one another. It is also clear that the diffusion is faster for larger values of $b$. In Fig. 4 we plot the energy $H(t)$ for four different values of the lattice coupling strength $b=0.002, b=0.02, b=0.2$, and $b=2.0$. In all four cases the initial energy is $E=30$, and the trajectory is run for a time $t=2000$. For each value of $b$, we have obtained energy plots for 10 different initial conditions on the energy surface. In Fig. 4 we show one realization (out of 10) for each value of $b$. As we can see from the plots, the energy fluctuates. We obtain the average energy for each plot, and then average the energy of the 10 plots for each value of $b$. We find the following average energies: $\left(b, E_{a v}\right)=$ $(0.002,35.8),(0.02,46.0),(0.2,79.0),(2.0,186.3)$. This change in the behavior of the average energy appears to be consistent with our estimates for the transition between the Nekhoroshev and Chirikov regimes. The Chirikov regime requires resonance overlap, which, for low values of $b$, will occur in local regions of the phase space because of the different sizes and locations of resonances. The behavior of the average energy indicates that large-scale diffusion in energy, due to resonance overlap, begins to occur for $b>0.2$ and steadily grows as $b$ increases.

In the next section, we will analyze the quantum dynamics of this system. We expect to find behavior analogous to what is seen in the classical system because the overlap of resonances (the Chirkov regime) also occurs in the quantum analog of this system. The effect of resonance overlap on quantum systems was analyzed in Refs. [11,18,19] for a system with $2 \mathrm{DoF}$, based on the fact that nonlinear resonances are pendulum-like structures and single pendulum is an integrable system whose quantum dynamics is governed by the Matheau equation. For the case when a resonance is large enough to hold a number of quantum states, semiclassical analysis can be used to analyze resonance overlap. However, regardless of the size of resonances, when they overlap "good" quantum numbers are destroyed and the wave function can spread through the resonance overlap region. In quantum systems, however, the extent of the spread of the wave function may be limited by dynamic Anderson localization. Detailed analysis of the overlap of specific resonances in systems with three or more DoF needs to done numerically $[8,9]$. In the subsequent sections, we focus on the global behavior 


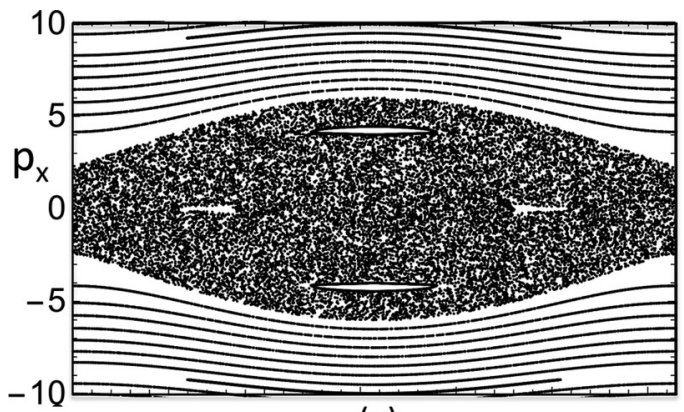

(a)

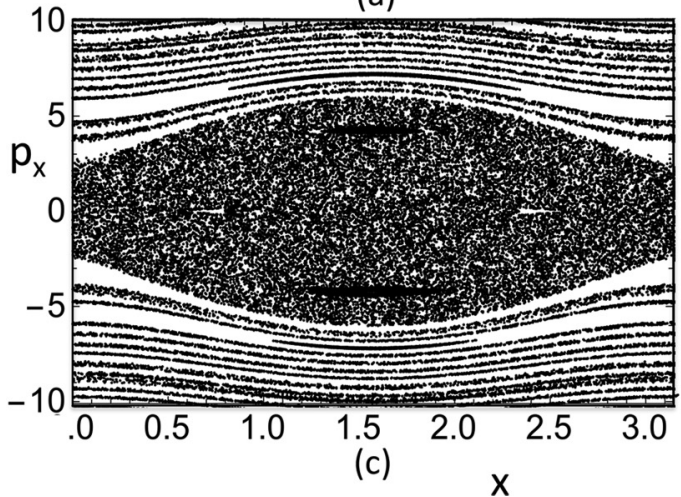

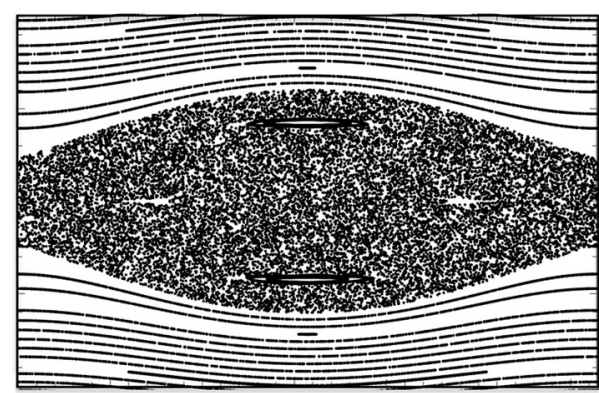

(b)

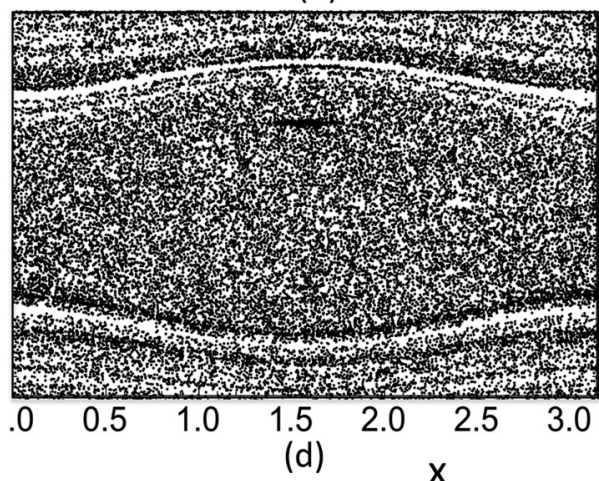

FIG. 3. Strobe plots of $p_{x}$ versus $x$ for initial conditions $x(0)=\frac{\pi}{2},-30 \leqslant p_{x}(0) \leqslant 30$ with spacing of $0.6, y(0)=\pi, p_{y}(0)=0.5$. (a) Strobe plot for sub-Hamiltonian $H_{x}=p_{x}^{2}+\frac{U}{2} \cos ^{2}(x)+\frac{U}{2} \cos (2 \omega t) \cos ^{2}(x)$. (b) Strobe plot for full Hamiltonian Eq. (5) for $b=0$. (c) Strobe plot for full Hamiltonian Eq. (5) for $b=0.002$. (d) Strobe plot for full Hamiltonian Eq. (5) for $b=0.02$.

of noninteracting atoms embedded in the time-periodically modulated two-dimensional optical lattice.

\section{QUANTUM DYNAMICS}

The quantum dynamics of particles in the optical lattice, in dimensionless units, is governed by the Schrödinger equation

$$
\begin{aligned}
i \frac{\partial}{\partial t} \psi(x, y, t)= & \left\{-\frac{\partial^{2}}{\partial x^{2}}-\frac{\partial^{2}}{\partial y^{2}}\right. \\
& \left.+\left[V_{0}+V_{1} \cos ^{2}(\omega t)\right] V(x, y)\right\} \psi(x, y, t),
\end{aligned}
$$
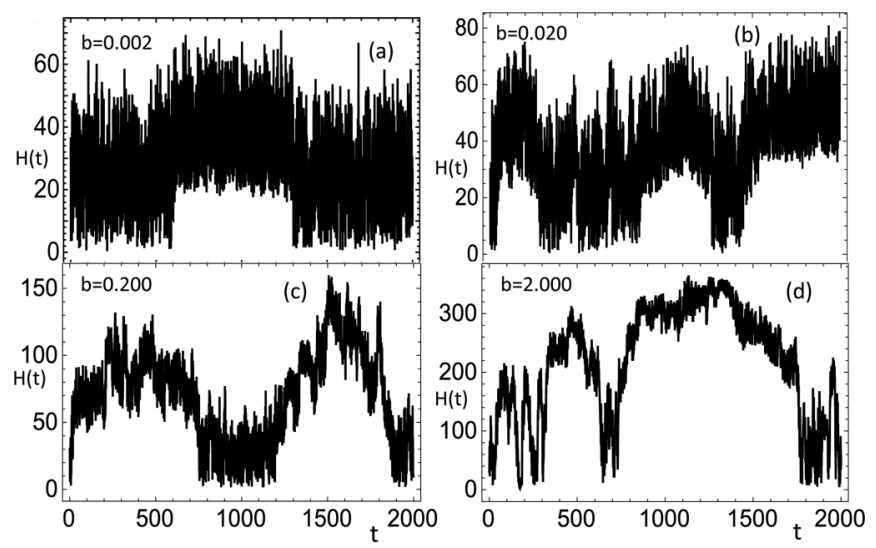

FIG. 4. Plots of $H(t)$, for a single trajectory, as a function of time for four different values of $b$. Each trajectory has initial energy $E=30$. (a) $b=0.002$; (b) $b=0.02$; (c) $b=0.2$; and (d) $b=2.0$. where $V(x, y)$ is given in Eq. (3). We will focus on the dynamics of a single unit cell of the optical lattice. Because the potential energy conserves parity in the $x$ and $y$ directions, the wave function $\psi(x, y, t)$ can be expanded in terms of functions that are either symmetric or antisymmetric under reflection through the origin. Each subspace is dynamically invariant because of symmetries and can be treated independently. A convenient set of basis functions is composed of sines and cosines with periodic boundary conditions on the unit cell. There will be invariant subspaces formed by each of the basis combinations $\cos \left(n_{x} x\right) \cos \left(n_{y} y\right), \sin \left(n_{x} x\right) \sin \left(n_{y} y\right)$, $\sin \left(n_{x} x\right) \cos \left(n_{y} y\right)$, and $\cos \left(n_{x} x\right) \sin \left(n_{y} y\right)$. In subsequent sections, we will focus on the subspace of the Hamiltonian formed by the basis set $\sin \left(n_{x} x\right) \sin \left(n_{y} y\right),\left(1 \leqslant n_{x} \leqslant \infty, 1 \leqslant n_{y} \leqslant \infty\right)$. The other blocks will behave in a qualitatively similar manner.

Before looking at the time-periodically driven system, it is useful to consider the time-independent case $V_{1}=0$. The Hamiltonian is

$$
\hat{H}^{(0)}=\hat{p}_{x}^{2}+\hat{p}_{y}^{2}+U\left[b \cos (\hat{x}) \cos (\hat{y})+\cos ^{2}(\hat{x})+\cos ^{2}(\hat{y})\right] .
$$

If we denote the $n$th eigenfunction of the Hamiltonian $\hat{H}^{(0)}$ as $\left|E_{n}\right\rangle$, then $\hat{H}^{(0)}\left|E_{n}\right\rangle=E_{n}\left|E_{n}\right\rangle$ and the solution to the Schrödinger equation can be written

$$
|\psi(t)\rangle=\mathrm{e}^{-i \hat{H}^{(0)} t}|\psi(0)\rangle=\sum_{n=1}^{\infty} \mathrm{e}^{-i E_{n} t}\left\langle E_{n} \mid \psi(0)\right\rangle\left|E_{n}\right\rangle .
$$

For the case $b=0.2$, the maximum value of the potential energy is $V_{\max }=44$, and there are nine antisymmetric energy eigenstates with energies below this value. They are plotted, 

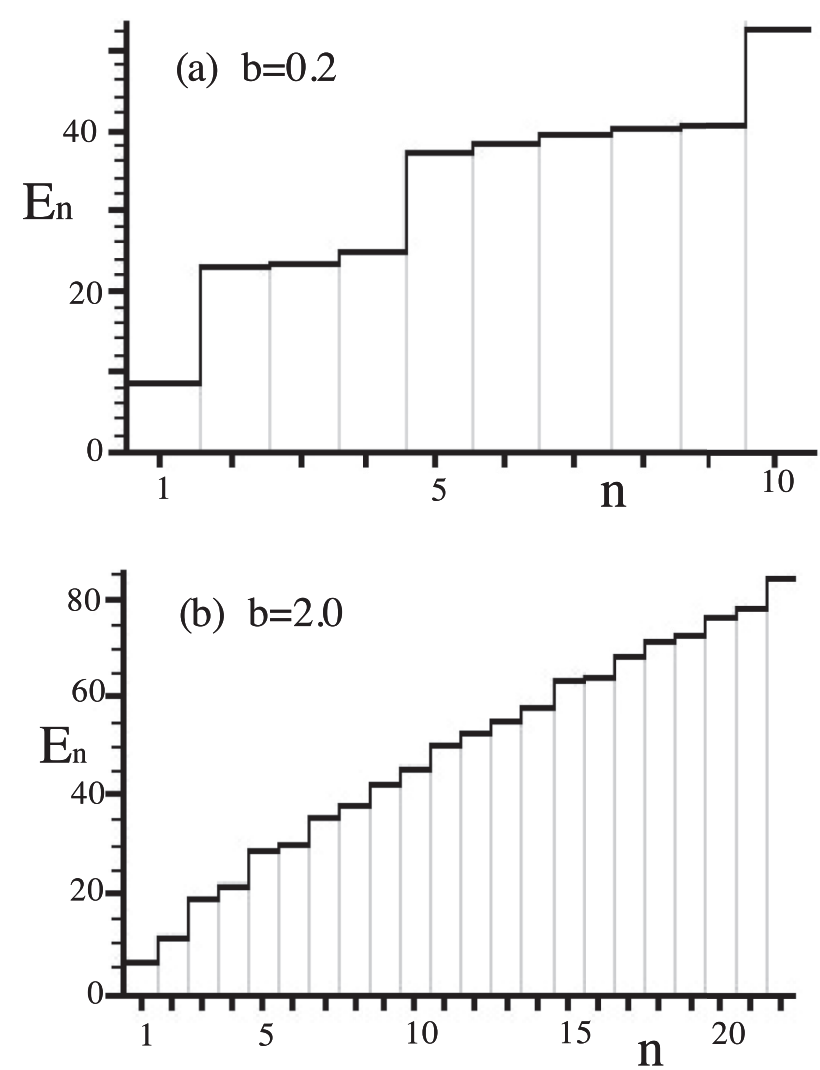

FIG. 5. Staircase function of energy eigenvalues of asymmetric energy eigenstates that lie below the potential energy maximum $V_{\max }$ for $V_{1}=0$. (a) $b=0.2$ and $V_{\max }=44$. (b) $b=2.0$ and $V_{\max }=80$. One state above the energy $E=V_{\max }$ is also included.

as a staircase function, in Fig. 5(a). For the case $b=2.0$, the maximum value of the potential energy is $V_{\max }=80$, and there are 21 antisymmetric energy eigenstates with energies below this value. They are plotted in Fig. 5(b).

We next consider the time-periodically driven system $\left(V_{1} \neq 0\right)$. Let us write the wave function in the form

$$
\psi(x, y, t)=\frac{1}{\pi} \sum_{n_{x}=1}^{\infty} \sum_{n_{y}=1}^{\infty} b_{n_{x}, n_{y}}(t) \sin \left(n_{x} x\right) \sin \left(n_{y} y\right) .
$$

If we substitute Eq. (16) into Eq. (13), we obtain

$$
i \frac{\partial}{\partial t} b_{m_{x}, m_{y}}(t)=\sum_{n_{x}=1}^{\infty} \sum_{n_{y}=1}^{\infty} H_{m_{x}, m_{y} ; n_{x}, n_{y}}(t) b_{n_{x}, n_{y}}(t)
$$

where

$$
H_{m_{x}, m_{y} ; n_{x}, n_{y}}(t)=\left(m_{x}^{2}+m_{y}^{2}\right) \delta_{m_{x}, n_{x}} \delta_{n_{y}, m_{y}}+V_{m_{x}, m_{y} ; n_{x}, n_{y}}(t)
$$

and

$$
\begin{aligned}
V_{m_{x}, m_{y} ; n_{x}, n_{y}}(t)= & \frac{1}{\pi^{2}} \int_{0}^{2 \pi} \int_{0}^{2 \pi} d x d y \sin \left(m_{x} x\right) \sin \left(m_{y} y\right) \\
& \times V(x, y, t) \sin \left(n_{x} x\right) \sin \left(n_{y} y\right) .
\end{aligned}
$$

For computational purposes, it is useful to separate the wave function into its real and imaginary parts. We let $b_{n_{x}, n_{y}}(t)=$ $p_{n_{x}, n_{y}}(t)+i q_{n_{x}, n_{y}}(t)$, and the Schrödinger equation then takes the form

$$
\frac{d}{d t} \Psi_{m_{x}, m_{y}}(t)=\sum_{n_{x}=1}^{\infty} \sum_{n_{y}=1}^{\infty} \mathbf{L}_{m_{x}, m_{y} ; n_{x}, n_{y}}(t) \Psi_{n_{x}, n_{y}}(t),
$$

where

$$
\Psi_{n_{x}, n_{y}}(t)=\left(\begin{array}{l}
p_{n_{x}, n_{y}}(t) \\
q_{n_{x}, n_{y}}(t)
\end{array}\right)
$$

and

$$
\mathbf{L}_{m_{x}, m_{y} ; n_{x}, n_{y}}(t)=\left(\begin{array}{cc}
0 & H_{m_{x}, m_{y} ; n_{x}, n_{y}}(t) \\
-H_{m_{x}, m_{y} ; n_{x}, n_{y}}(t) & 0
\end{array}\right) .
$$

In practice it is necessary to truncate the infinite Hamiltonian matrix to a finite size. We can check the reliability of our results by computing eigenvalues for different size matrices, and then keep those that don't change as we change the size of the matrix. Generally the lowest energy eigenvalues are the ones that are most stable to change in matrix size.

Let us now introduce the $1 \times N^{2}$ column matrices $\overline{\mathbf{p}}$ and $\overline{\mathbf{q}}$, which are defined (we write the transpose)

$$
\begin{aligned}
\overline{\mathbf{p}}^{T}= & \left(p_{1,1}, p_{1,2}, \ldots, p_{1, N}, p_{2,1}, \ldots p_{2, N}, p_{3,1}, \ldots, p_{N, N}\right) \\
\text { and } \quad \overline{\mathbf{q}}^{T}= & \left(q_{1,1}, q_{1,2}, \ldots, q_{1, N}, q_{2,1}, \ldots q_{2, N}\right. \\
& \left.\times q_{3,1}, \ldots, q_{N, N}\right)
\end{aligned}
$$

such that $\overline{\mathbf{b}}=\overline{\mathbf{p}}+i \overline{\mathbf{q}}$. Orthonormality of the wave function requires that $\overline{\mathbf{b}}^{\dagger} \cdot \overline{\mathbf{b}}=1$. If the $1 \times 2 N^{2}$ column matrix $\overline{\bar{\Psi}}$ is defined

$$
\overline{\bar{\Psi}}(t)=\left(\begin{array}{l}
\overline{\mathbf{p}}(t) \\
\overline{\mathbf{q}}(t)
\end{array}\right)
$$

then the equation of motion of $\overline{\bar{\Psi}}(t)$ is given by

$$
\frac{d}{d t} \overline{\bar{\Psi}}(t)=\overline{\overline{\mathbf{L}}}(t) \cdot \overline{\bar{\Psi}}(t),
$$

where $\overline{\overline{\mathbf{L}}}(t)$ is the real skew-symmetric $2 N^{2} \times 2 N^{2}$ matrix

$$
\overline{\overline{\mathbf{L}}}(t)=\left(\begin{array}{cc}
0 & \overline{\mathbf{H}}(t) \\
-\overline{\mathbf{H}}(t) & 0
\end{array}\right) .
$$

Note that $\overline{\overline{\mathbf{L}}}(t)$ is periodic in time with period $T_{0}=\frac{2 \pi}{\omega}$. In terms of the column matrices $\overline{\mathbf{p}}(t)$ and $\overline{\mathbf{q}}(t)$, the Schrödinger equation can be written in terms of the coupled equations

$$
\frac{d \overline{\mathbf{p}}(t)}{d t}=\overline{\mathbf{H}}(t) \cdot \overline{\mathbf{q}}(t) \text { and } \frac{d \overline{\mathbf{q}}(t)}{d t}=-\overline{\mathbf{H}}(t) \cdot \overline{\mathbf{p}}(t) .
$$

We have now reduced the quantum problem to the task of solving $2 \mathrm{~N}$ coupled first order differential equations with timeperiodic coefficients. For this we need Floquet theory.

\section{FLOQUET STATES}

Let us consider the equation of motion (25) where $\overline{\overline{\mathbf{L}}}(t)$ is time-periodic with period $T_{0}=\frac{2 \pi}{\omega}$. Assume that Eq. (25) has a Floquet-type solution of the form

$$
\left(\begin{array}{c}
\overline{\mathbf{p}}_{\alpha}(t) \\
\overline{\mathbf{q}}_{\alpha}(t)
\end{array}\right)=\mathrm{e}^{i \Omega_{\alpha} t} \Phi_{\alpha}(t) \equiv \mathrm{e}^{i \Omega_{\alpha} t}\left(\begin{array}{c}
\overline{\mathcal{P}}_{\alpha}(t) \\
\overline{\mathcal{Q}}_{\alpha}(t)
\end{array}\right),
$$


where $\Omega_{\alpha}$ is the $\alpha$ th Floquet eigenphase (also called quasienergy) and the Floquet eigenstate, $\Phi^{T}(t)=\left(\overline{\mathcal{P}}_{\alpha}(t), \overline{\mathcal{Q}}_{\alpha}(t)\right)^{T}, \quad$ is periodic with period $T_{0}$ :

$$
\left(\begin{array}{l}
\overline{\mathcal{P}}_{\alpha}\left(T_{0}\right) \\
\overline{\mathcal{Q}}_{\alpha}\left(T_{0}\right)
\end{array}\right)=\left(\begin{array}{c}
\overline{\mathcal{P}}_{\alpha}(0) \\
\overline{\mathcal{Q}}_{\alpha}(0)
\end{array}\right)
$$

The Floquet eigenstate satisfies the eigenvalue equation

$$
\overline{\overline{\mathcal{L}}}_{\mathcal{F}} \cdot\left(\begin{array}{c}
\overline{\mathcal{P}}_{\alpha}(t) \\
\overline{\mathcal{Q}}_{\alpha}(t)
\end{array}\right)=i \Omega_{\alpha}\left(\begin{array}{c}
\overline{\mathcal{P}}_{\alpha}(t) \\
\overline{\mathcal{Q}}_{\alpha}(t)
\end{array}\right)
$$

where the Floquet operator, $\overline{\overline{\mathcal{L}}}_{\mathcal{F}}$, is defined $\overline{\overline{\mathcal{L}}}_{\mathcal{F}}=\left(\overline{\overline{\mathbf{L}}}(t)-\frac{d}{d t}\right)$. The solution to the Schrödinger equation can be expanded in a complete set of Floquet eigenstates so that

$$
\left(\begin{array}{l}
\overline{\mathbf{p}}(t) \\
\overline{\mathbf{q}}(t)
\end{array}\right)=\sum_{\alpha} A_{\alpha} \mathrm{e}^{i \Omega_{\alpha} t}\left(\begin{array}{c}
\overline{\mathcal{P}}_{\alpha}(t) \\
\overline{\mathcal{Q}}_{\alpha}(t)
\end{array}\right) .
$$

Since

$$
\left(\begin{array}{l}
\overline{\mathbf{p}}(0) \\
\overline{\mathbf{q}}(0)
\end{array}\right)=\sum_{\alpha} A_{\alpha}\left(\begin{array}{c}
\overline{\mathcal{P}}_{\alpha}(0) \\
\overline{\mathcal{Q}}_{\alpha}(0)
\end{array}\right)
$$

and the Floquet eigenstates are assumed to be othonormal so

$$
\left(\overline{\mathcal{P}}_{\alpha^{\prime}}^{\dagger}(0), \quad \overline{\mathcal{Q}}_{\alpha^{\prime}}^{\dagger}(0)\right)^{\dagger} \cdot\left(\begin{array}{c}
\overline{\mathcal{P}}_{\alpha}(0) \\
\overline{\mathcal{Q}}_{\alpha}(0)
\end{array}\right)=\delta_{\alpha^{\prime}, \alpha},
$$

the coefficients $A_{\alpha}$ can be written

$$
A_{\alpha}=\left(\overline{\mathcal{P}}_{\alpha}^{\dagger}(0), \quad \overline{\mathcal{Q}}_{\alpha}^{\dagger}(0)\right)^{\dagger} \cdot\left(\begin{array}{c}
\overline{\mathbf{p}}(0) \\
\overline{\mathbf{q}}(0)
\end{array}\right) .
$$

The solution then takes the form

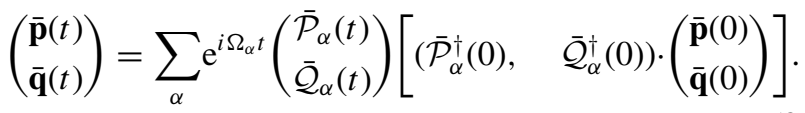

At time $t=T_{0}$ we can write

$$
\left(\begin{array}{l}
\overline{\mathbf{p}}\left(T_{0}\right) \\
\overline{\mathbf{q}}\left(T_{0}\right)
\end{array}\right)=\overline{\overline{\mathcal{U}}}\left(T_{0}\right) \cdot\left(\begin{array}{l}
\overline{\mathbf{p}}(0) \\
\overline{\mathbf{q}}(0)
\end{array}\right)
$$

where

$$
\overline{\overline{\mathcal{U}}}\left(T_{0}\right)=\sum_{\alpha} \mathrm{e}^{i \Omega_{\alpha} T_{0}}\left(\begin{array}{c}
\overline{\mathcal{P}}_{\alpha}(0) \\
\overline{\mathcal{Q}}_{\alpha}(0)
\end{array}\right)\left(\overline{\mathcal{P}}_{\alpha}^{\dagger}(0), \quad \overline{\mathcal{Q}}_{\alpha}^{\dagger}(0)\right)
$$

is the unitary Floquet evolution matrix with eigenvalues $\mathrm{e}^{i \Omega_{\alpha} T_{0}}$.

The solution to the Schrödinger equation at time $n T_{0}$ is

$$
\left(\begin{array}{l}
\overline{\mathbf{p}}\left(n T_{0}\right) \\
\overline{\mathbf{q}}\left(n T_{0}\right)
\end{array}\right)=\left(\overline{\mathcal{U}}\left(T_{0}\right)\right)^{n} \cdot\left(\begin{array}{c}
\overline{\mathbf{p}}(0) \\
\overline{\mathbf{q}}(0)
\end{array}\right) .
$$

The spatial distribution of the $\alpha$ th Floquet eigenstate is given by

$$
\begin{aligned}
\Phi_{\alpha}(x, y)= & \frac{1}{\pi} \sum_{n_{x}=1}^{\infty} \sum_{n_{y}=1}^{\infty}\left(\overline{\mathcal{P}}_{\alpha}(0)_{n_{x}, n_{y}}\right. \\
& \left.+i \overline{\mathcal{Q}}_{\alpha}(0)_{n_{x}, n_{y}}\right) \sin \left(n_{x} x\right) \sin \left(n_{y} y\right) .
\end{aligned}
$$

\section{A. Behavior of quantum states}

We can follow the behavior of the quantum states as we turn on the amplitude $V_{1}$ of the TPM of the optical lattice. For the case $V_{1}=0$, the Hamiltonian is independent of time, and we can write

$$
\overline{\mathbf{L}}^{(0)} \equiv\left(\begin{array}{cc}
0 & \overline{\mathbf{H}}^{(0)} \\
-\overline{\mathbf{H}}^{(0)} & 0
\end{array}\right) .
$$

The solution to the Schrödinger equation (25) can be written

$$
\overline{\bar{\Phi}}(t)=\mathrm{e}^{\overline{\mathbf{L}}^{(0)} t} \overline{\bar{\Phi}}(0) .
$$

We denote the $n$th and $(n+1)$ th eigenvectors of $L$ as

$$
\bar{\phi}_{2 n-1}=\frac{1}{\sqrt{2}}\left(\begin{array}{c}
i\left|E_{n}\right\rangle \\
\left|E_{n}\right\rangle
\end{array}\right) \text { and } \bar{\phi}_{2 n}=\frac{1}{\sqrt{2}}\left(\begin{array}{c}
-i\left|E_{n}\right\rangle \\
\left|E_{n}\right\rangle
\end{array}\right) \text {. }
$$

Then $\overline{\overline{\mathbf{L}}}^{(0)} \bar{\phi}_{2 n-1}=-i E_{n} \bar{\phi}_{2 n-1}$ and $\overline{\overline{\mathbf{L}}}^{(0)} \bar{\phi}_{2 n}=i E_{n} \bar{\phi}_{2 n}$. Furthermore, $\bar{\phi}_{2 n-1}=\bar{\phi}_{2 n}^{*}$. For $V_{1}=0$, the Floquet eigenphases occur in pairs that are equal to $\pm i E_{n}$, and the real and imaginary parts of the Floquet eigenstates are equal to the energy eigenstates. Therefore, at $V_{1}=0$ we can identify each Floquet eigenphase and eigenstate with an energy eigenvalue and eigenstate. Then, as we turn on the modulation, we can follow how each Floquet state changes from a pure energy eigenstate to an energy entangled state as the amplitude of the TPM increases. We do this by checking the overlap (orthonormality) of Floquet
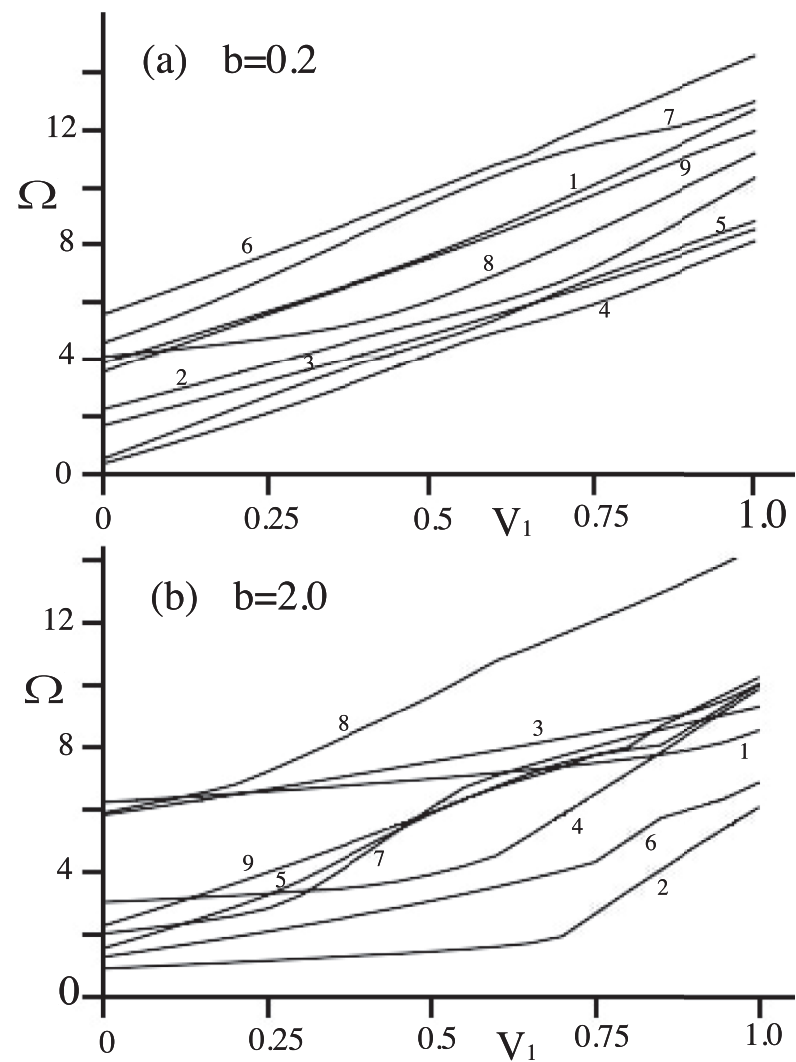

FIG. 6. Floquet eigenphases as a function of time-periodic modulation amplitude $V_{1}$. For $V_{1}=0, \Omega_{\alpha}=E_{n} \delta_{\alpha, n}(\bmod 2 \pi)$. The change in $\Omega_{\alpha}$ for $\alpha=1, \ldots, 9$ and for $0 \leqslant V_{1} \leqslant 1.0$ is shown (states are labeled with value of $\alpha$ ). (a) $b=0.2$; (b) $b=2.0$. 

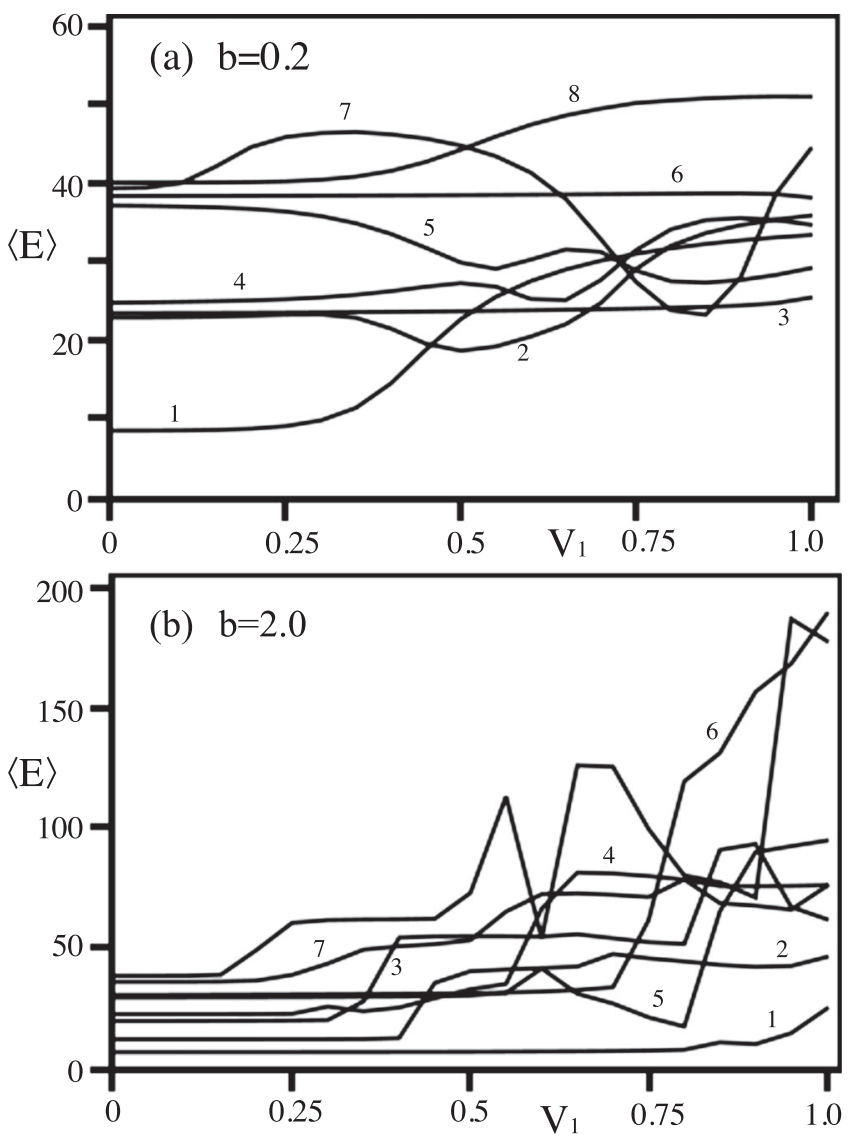

FIG. 7. Change in the average energy $\langle E\rangle=\left\langle\Phi_{\alpha}|\hat{H}| \Phi_{\alpha}\right\rangle$ of the Floquet eigenstates for $\alpha=1, \ldots, 8$, as a function of $V_{1}$ (states are labeled with value of $\alpha$ ). (a) $b=0.2$; (b) $b=2.0$. For $V_{1}=0$, $\left|\Phi_{\alpha=n}\right\rangle=\left|E_{n}\right\rangle$.

$(b=0.2)$
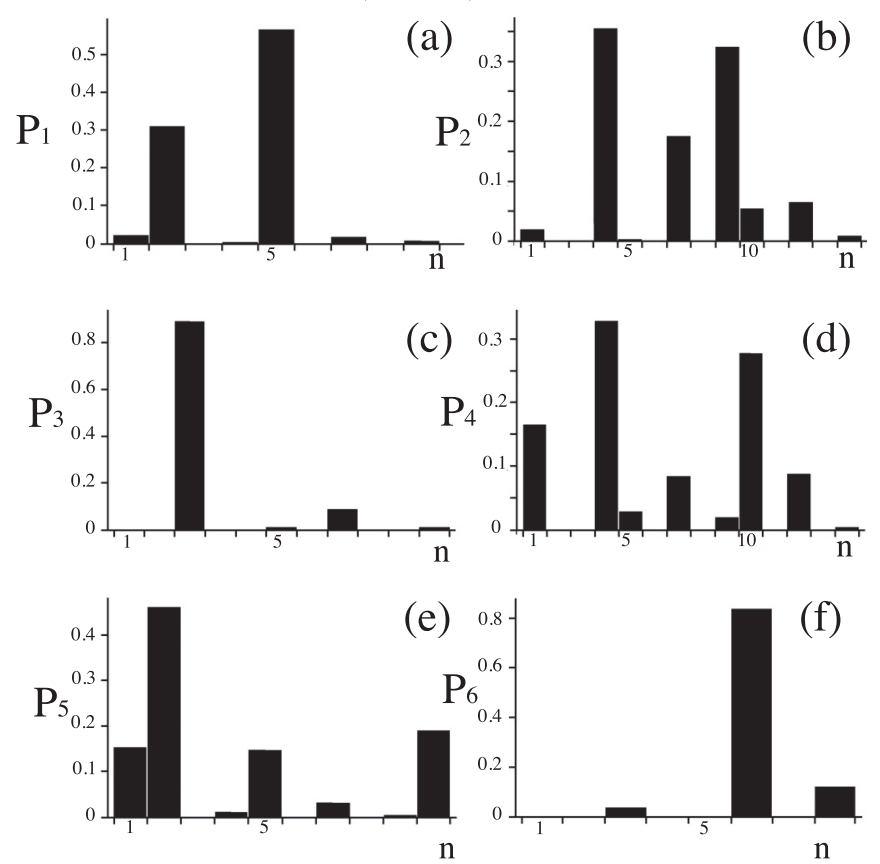

FIG. 8. The overlap probabilities $P_{\alpha}=\left|\left\langle E_{n} \mid \Phi_{\alpha}\right\rangle\right|^{2}$ for $n=1, \ldots$, $b=0.2, V_{1}=1.0$ and (a) $\alpha=1$; (b) $\alpha=2$; (c) $\alpha=3$; (d) $\alpha=4$; (e) $\alpha=5$; (f) $\alpha=6$. Note that, for $V_{1}=0, P_{\alpha}=\left|\left\langle E_{n} \mid \Phi_{\alpha}\right\rangle\right|^{2}=\delta_{\alpha, n}$.

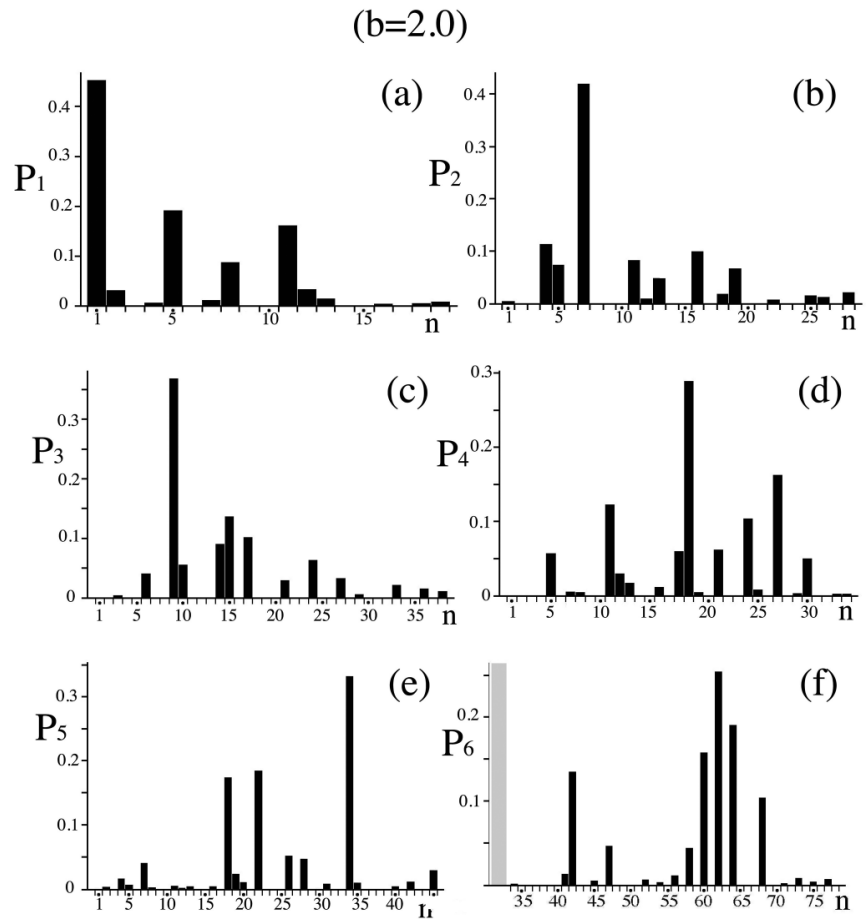

FIG. 9. The overlap probabilities $P_{\alpha}=\left|\left\langle E_{n} \mid \Phi_{\alpha}\right\rangle\right|^{2}$ for $n=1, \ldots$, $b=2.0, V_{1}=1.0$ and (a) $\alpha=1$; (b) $\alpha=2$; (c) $\alpha=3$; (d) $\alpha=4$; (e) $\alpha=5$; (f) $\alpha=6$. Note that, for $V_{1}=0, P_{\alpha}=\left|\left\langle E_{n} \mid \Phi_{\alpha}\right\rangle\right|^{2}=\delta_{\alpha, n}$.

states, $\bar{\Phi}_{\alpha}^{T}\left(V_{1}\right) \cdot \bar{\Phi}_{\alpha^{\prime}}\left(V_{1}+\epsilon\right)$, for $\epsilon \leqslant 0.05$ and identifying each pair of states whose overlap is greater than $90 \%$.

The Floquet eigenphases are only defined modulus $\omega$. For all data considered here, $\omega=2 \pi$. In Fig. 6 we plot nine Floquet eigenphases as a function of $V_{1}$. We have selected those eigenphases whose eigenstates correspond to the nine lowest energy eigenstates for $V_{1}=0$. These nine Floquet eigenphases are equal to the energy eigenvalues, modulus $2 \pi$. We have folded all nine Floquet eigenphases into the interval $0 \leqslant \Omega \leqslant 2 \pi$ for $V_{1}=0$. Then, as we increase $V_{1}$ we have let them "unfold" outside the interval $0 \leqslant \Omega \leqslant 2 \pi$ for visualization purposes. In Fig. 6(a) we have plotted the nine Floquet eigenphases for $b=0.2$. We see that they evolve fairly smoothly with increasing $V_{1}$, with only a few apparent avoided crossings. In Fig. 6(b) we have plotted the nine Floquet eigenphases for $b=2.0$. In this case, the evolution of the eigenphases is very erratic with a number of avoided crossings apparent, some even with states not shown in the plot. The avoided crossings are an indication of broken symmetries and the onset of large-scale chaos in the underlying classical system.

In Fig. 7 we show the average energy $\langle E\rangle=\bar{\Phi}_{\alpha}^{T} \cdot \bar{L}^{0} \cdot \bar{\Phi}_{\alpha}^{T}$ for the Floquet eigenstates $\alpha=1, \ldots, 8$. At $V_{1}=0$, the average energy is equal to the energy eigenvalue $E_{n=\alpha}$ and remains essentially unchanged for small nonzero values of $V_{1}$. However, for larger, nonzero values of $V_{1}$ the average energy undergoes significant excursions, indicating that the Floquet eigenstates begin to consist of a superposition of a number of energy eigenstates. In Fig. 7(a) we show the average energy of Floquet eigenstates $\alpha=1, \ldots, 8$ for $b=0.2$. There is a slight increase of the average energy of some states as $V_{1}$ 


\section{$\mathrm{b}=0.2$}

(a) $\alpha=1, V_{1}=0$

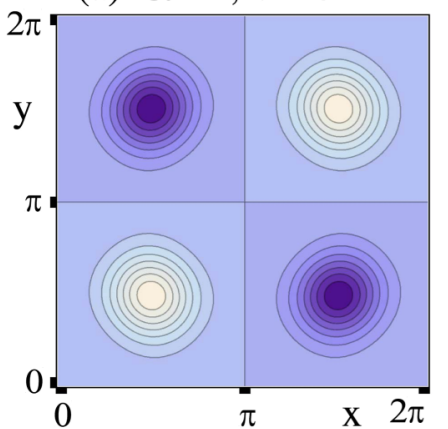

(c) $\alpha=2, V_{1}=0$

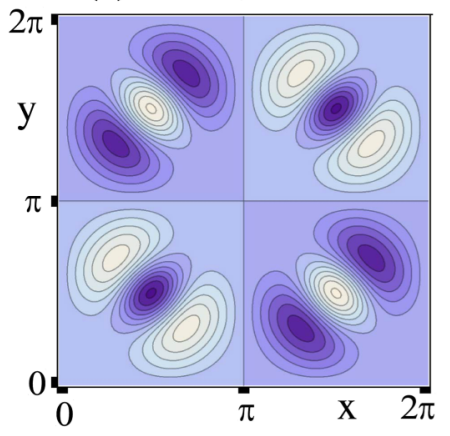

(e) $\alpha=6, V_{1}=0$

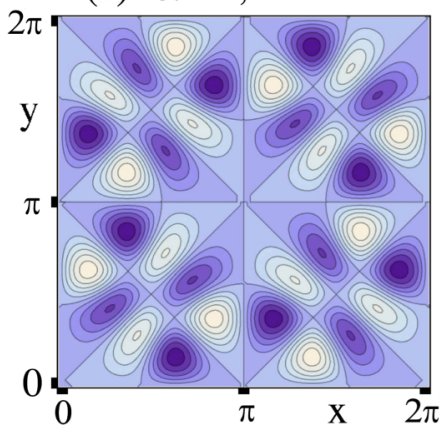

(b) $\alpha=1, V_{1}=1.0$

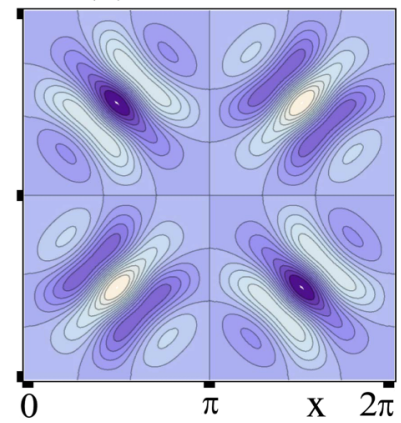

(d) $\alpha=2, V_{1}=1.0$

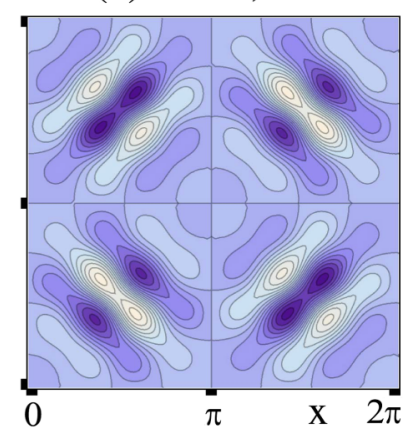

(f) $\alpha=6, V_{1}=1.0$

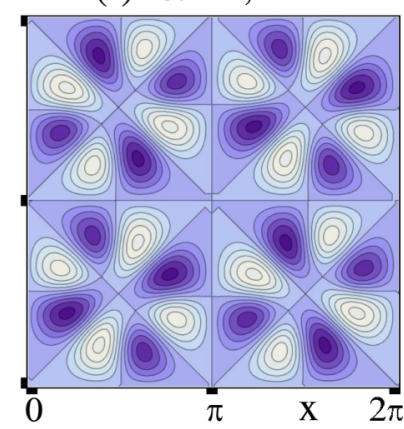

FIG. 10. Floquet eigenstates on the unit cell for $b=0.2$. (a) $\alpha=$ $1, V_{1}=0$. (b) $\alpha=1, V_{1}=1.0$. (c) $\alpha=2, V_{1}=0$. (d) $\alpha=2, V_{1}=$ 1.0. (e) $\alpha=6, V_{1}=0$. (f) $\alpha=6, V_{1}=1.0$.

increases, but not a significant increase. Two of the states, $\alpha=3$ and $\alpha=6$, are almost completely unchanged by the TPM. In Fig. 7(b) we show the average energy of Floquet eigenstates $\alpha=1, \ldots, 8$ for $b=2.0$. The average energy now takes significant excursions well above the maximum potential energy for that value of coupling parameter, $b$. The average energy of the state $\alpha=6$ now gets "pumped" to very high energy. This growth in the average energy is consistent with the classical behavior of the average energy. We expect that the growth in average energy will lag the classical system (as a function of $b$ ) because the quantum system does not "see" phase space structure smaller than $\hbar$. However, for $b=2$, the classical and quantum results for average energy are in good agreement.

In Figs. 8 and 9 we show the overlap probability $P_{\alpha}=$ $\left|\bar{\Psi}_{n}^{T} \cdot \bar{\Phi}_{\alpha}\right|^{2}$, to find the $\alpha$ th Floquet eigenstate in the $n$th energy state for $V_{1}=1$. Note that $P_{\alpha}=\left|\bar{\Psi}_{n}^{T} \cdot \bar{\Phi}_{\alpha}\right|^{2}=\delta_{n, \alpha}$ for $V_{1}=0$. Figure 8 (a) shows the overlap probability for states

\section{$b=2.0$}

(a) $\alpha=1, V_{1}=0$

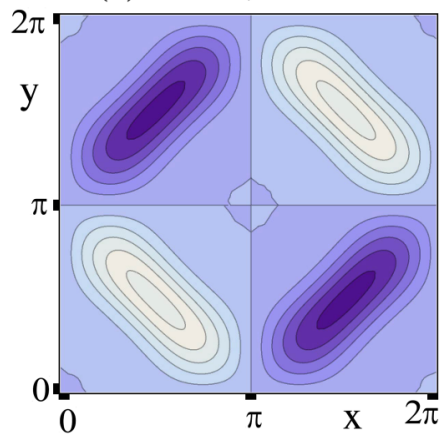

(c) $\alpha=2, V_{1}=0$

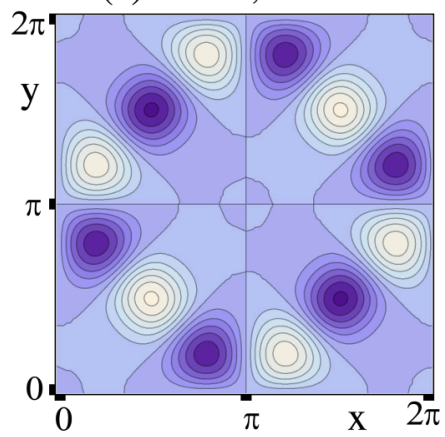

(e) $\alpha=6, V_{1}=0$

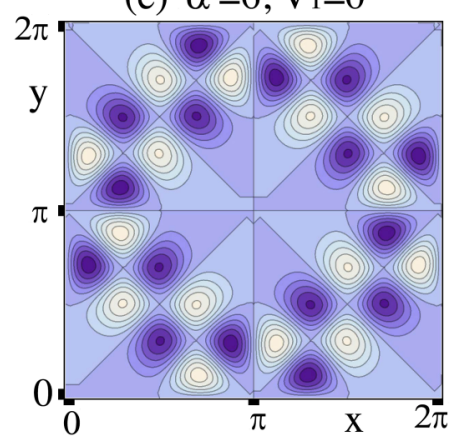

(b) $\alpha=1, V_{1}=1.0$

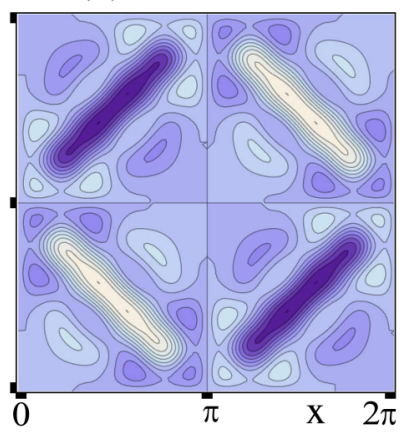

(d) $\alpha=2, V_{1}=1.0$

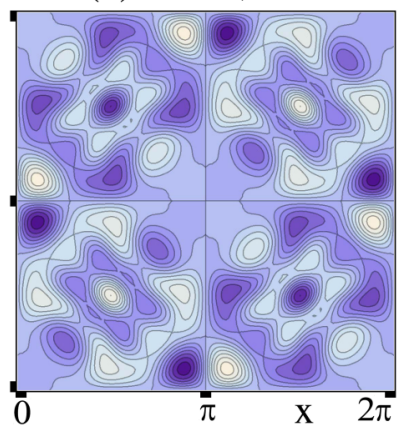

(f) $\alpha=6, V_{1}=1.0$

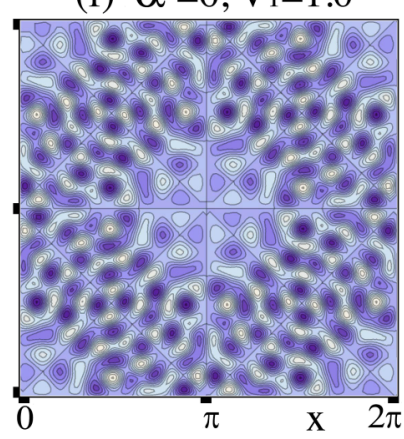

FIG. 11. Floquet eigenstates on the unit cell for $b=2.0$. (a) $\alpha=$ $1, V_{1}=0$; (b) $\alpha=1, V_{1}=1.0$; (c) $\alpha=2, V_{1}=0$; (d) $\alpha=2, V_{1}=$ 1.0 ; (e) $\alpha=6, V_{1}=0$; (f) $\alpha=6, V_{1}=1.0$.

$\alpha=1, \ldots, 6$ for $b=0.2$. Comparison of these plots with the corresponding average energy plots in Fig. 7(a) shows qualitative agreement in the behavior of the two kinds of plots. For example, in Fig. 7(a) the average energy of states $\alpha=3$ and $\alpha=6$ remains very little changed from their energy at $V_{1}=0$, while in Figs. 8(c) and 8(f) their overlap probability with energy states $n=3$ and $n=6$, respectively, is above 80\%. In Fig. 8(b) we show the overlap probabilities $P_{\alpha}$ for $\alpha=1, \ldots, 6$ for $b=2.0$. These overlap probabilities again agree qualitatively with the average energy plots. Note that for $b=2.0$, the states $\alpha=3$ and $\alpha=6$ consist of a superposition of very high energy eigenstates. The state $\alpha=6$, whose average energy in Fig. 7(b) goes to very high energy, no longer has any overlap with energy states below the potential energy maximum in Fig. 8(f).

Finally, in Figs. 10 and 11, we show the spatial distribution of the Floquet states $\alpha=1,2,6$ in the unit cell for $b=0.2$ 
and $b=2.0$, respectively. In both figures we show the state at $V_{1}=0$, when it is an energy eigenstate, and at $V_{1}=1.0$, when it is maximally driven system. In Fig. 10 where $b=0.2$, we see that the states $\alpha=1,2$ are significantly changed as the modulation amplitude goes from $V_{1}=0$ to $V_{1}=1.0$, and the Floquet state becomes an entangled collection of energy eigenstates. However, only minimal changes occur in the state $\alpha=6$. In Fig. 11 where $b=2.0$, we see that the states $\alpha=1,2$ are again significantly changed as the modulation amplitude goes from $V_{1}=0$ to $V_{1}=1.0$. However, the state $\alpha=6$ is completely changed and appears to lie in a chaotic sea.

\section{CONCLUSIONS}

The effect of time-periodic forces on material systems is a topic of growing importance, especially in view of the growing use of lasers to control transitions in molecular and solid state systems. Optical lattices provide an ideal medium for studying the dynamics of the laser-matter interaction in complex systems, because the theoretical analysis is simple enough that it allows detailed analysis of the dynamics.

We have analyzed both the classical and quantum dynamics of a 2 DoF optical lattice whose amplitude undergoes a TPM. The TPM increases the number of DoF to $2.5 \mathrm{DoF}$, and we find that an Arnold web, consisting of a dense set of resonance lines, forms in the phase space of the 2.5 DoF optical lattice causing the system to become intrinsically unstable. As we increase the strength of the coupling between the static DoF, the width of the resonance lines in the Arnold web increases and the degree of overlap increases, allowing more rapid large-scale diffusion throughout the phase space.

In conservative systems with three or more DoF, energy is conserved and the rate at which the system diffuses throughout the phase space can be controlled if coupling between DoF can be controlled. However, Arnold diffusion in time-periodically driven systems can be particularly troublesome because energy is not conserved, and Arnold diffusion can occur over large ranges of energy. Indeed, we have found that Arnold diffusion occurs in both the classical and quantum dynamics of the 2.5 DoF optical lattice. It causes large random excursions in energy in the classical dynamics of the lattice, and it gives rise to Floquet eigenstates consisting of large numbers of entangled energy states in the quantum system. This energy instability is not limited to driven optical lattices, but is a universal property of any driven material system with 2.5 or more DoF.

\section{ACKNOWLEDGMENTS}

The authors thank the Robert A. Welch Foundation (USA), F-1051 for support of this work. L.E.R. thanks Dan Steck for very helpful comments regarding experimental realization of modulated optical lattices. Both authors thank Christof Jung for helpful discussions concerning the lattice dynamics.

\section{APPENDIX A}

The Hamiltonian for the center of mass motion of twolevel atoms in an optical lattice with electric field $\mathbf{E}(x, y, t)=$
$\mathbf{A}(x, y, t) \mathrm{e}^{-i \omega_{L} t}+\mathbf{A}^{*}(x, y, t) \mathrm{e}^{+i \omega_{L} t}$ can be written $[32,33]$

$$
H_{S I}=\frac{p_{x}^{2}}{2 m}+\frac{p_{y}^{2}}{2 m}-\frac{d^{2}|\mathbf{A}(x, y, t)|^{2}}{\hbar \Delta},
$$

where $m$ is the mass of the particles and $\Delta$ is the detuning of the radiation field from resonance with the two-level atoms. We start with an electric field that consists of four pairs of oppositely traveling waves, two pairs in the $x$ direction and two pairs in the $y$ direction. The waves traveling in the $x$ direction ( $y$ direction) have polarization unit vector $\hat{\epsilon}_{1}\left(\hat{\epsilon}_{2}\right)$. Each pair has different frequency and wave vector (shifted by an AOM by a small amount). The electric field takes the form

$$
\begin{aligned}
E(x, y, t)= & \hat{\epsilon}_{1} \cos \left(k_{L, 1} x+\omega_{L, 1} t\right)+\hat{\epsilon}_{1} \cos \left(k_{L, 1} x-\omega_{L, 1} t\right) \\
& +\hat{\epsilon}_{1} \cos \left(k_{L, 2} x+\omega_{L, 2} t\right)+\hat{\epsilon}_{1} \cos \left(k_{L, 2} x-\omega_{L, 2} t\right) \\
& \left.+\hat{\epsilon}_{2} \cos k_{L, 1} y+\omega_{L, 1} t\right)+\hat{\epsilon}_{2} \cos \left(k_{L, 1} y-\omega_{L, 1} t\right) \\
& +\hat{\epsilon}_{2} \cos \left(k_{L, 2} y+\omega_{L, 2} t\right)+\hat{\epsilon}_{2} \cos \left(k_{L, 2} y-\omega_{L, 2} t\right) .
\end{aligned}
$$

The wave vectors and frequencies can be written

$$
\begin{aligned}
k_{L, 1} & =k_{L}+\delta k, \quad k_{L, 2}=k_{L}-\delta k, \quad \omega_{L, 1}=\omega_{L}+\delta \omega, \\
\omega_{L, 2} & =\omega_{L}-\delta \omega,
\end{aligned}
$$

where $\delta k \ll k_{L}$ and $\delta \omega \ll \omega_{L}$.

We now write the electric field in the form $E(x, y, t)=$ $A(x, y, t) \mathrm{e}^{-i \omega_{L} t}+A^{*}(x, y, t) \mathrm{e}^{+i \omega_{L} t}$. If we substitute Eq. (A3) into the equation for $A(x, t)$, set $\delta k=0$ (remove very long wavelength corrections that do not significantly affect the dynamics), and remove the terms that depend on frequency $\delta \omega$ without any associated space dependence, we obtain

$$
\begin{aligned}
|A(x, y, t)|^{2}= & \left|E_{0}\right|^{2}\left\{\left(2+4 \cos ^{2}(\delta \omega t)\left[\cos ^{2}\left(k_{L} x\right)+\cos ^{2}\left(k_{L} y\right)\right.\right.\right. \\
& \left.\left.+2 \hat{\epsilon}_{1} \cdot \hat{\epsilon}_{2} \cos \left(k_{L} x\right) \cos \left(k_{L} y\right)\right]\right\} .
\end{aligned}
$$

(These approximations were used to obtain the Hamiltonians describing the experiments [25,26,28], and they give remarkably good agreement with experimental results [27].) Note that we have also used the relation $\cos \left(2 k_{L} x\right)=2 \cos ^{2}\left(k_{L} x\right)-1$. The Hamiltonian then takes the form

$$
\begin{aligned}
H_{S I}= & \frac{p_{x}^{2}}{2 m}+\frac{p_{y}^{2}}{2 m}-\frac{2 d^{2} E_{0}^{2}}{\hbar \Delta}\left\{1+2 \cos ^{2}(\delta \omega t)\left[\cos ^{2}\left(k_{L} x\right)\right.\right. \\
& \left.\left.+\cos ^{2}\left(k_{L} y\right)+2 \hat{\epsilon}_{1} \cdot \hat{\epsilon}_{2} \cos \left(k_{L} x\right) \cos \left(k_{L} y\right)\right]\right\} .
\end{aligned}
$$

Now go to dimensionless units. Let $E_{L}=\frac{\hbar^{2} k_{L}^{2}}{2 m}$ and $\omega_{L}=$ $\frac{\hbar k_{L}^{2}}{2 m}$. Let $x^{\prime}=k_{L} x, y^{\prime}=k_{L} y, H^{\prime}=H / E_{L}, t^{\prime}=\omega_{L} t, \delta \omega=$ $\omega^{\prime} \omega_{L}$, and $U=-\frac{4 d^{2} E_{o}^{2}}{E_{L} \hbar \Delta}$ (we can change the sign of $U$ by changing the detuning parameter). The primed quantities are dimensionless. Then drop the primes to get

$$
\begin{aligned}
H= & p_{x}^{2}+p_{y}^{2}+U \cos ^{2}(\omega t)\left\{\left[\cos ^{2}(x)+\cos ^{2}(y)\right]\right. \\
& +b \cos (x) \cos (y)\}+\frac{U}{2},
\end{aligned}
$$

where $b=2 \hat{\epsilon}_{1} \cdot \hat{\epsilon}_{2}$. It is useful to note that the dynamics described by this Hamiltonian remains invariant under the scaling $t \rightarrow t / a, \omega \rightarrow a \omega, p \rightarrow p / a, U \rightarrow a^{2} U$, and $H \rightarrow a^{2} H$. In this paper, our Hamiltonian is scaled to be consistent with the experiments in Refs. [25,26]. 


\section{APPENDIX B}

Below we list the canonical transformation for the libration region and rotation regions of the pendulum Hamiltonian [11]

$$
H_{x}=p_{x}^{2}+V \cos ^{2}(x)=E_{x} .
$$

Libration occurs for $E_{x}<V$. Rotation occurs for $E_{x}>V$.

\section{Action-angle variables: Libration}

For energies $E_{x}<V$, the pendulum liberates and the action variable is defined

$$
J_{x}=\frac{2 \sqrt{V}}{\pi}\left[\mathrm{E}\left(\kappa_{x}\right)-\left(1-\kappa^{2}\right) \mathrm{K}\left(\kappa_{x}\right)\right],
$$

where $\mathrm{K}\left(\kappa_{x}\right)$ is the complete elliptic integral of the first kind, $\mathrm{E}\left(\kappa_{x}\right)$ is the complete elliptic integral of the second kind, and the modulus is $\kappa_{x}^{2}=E_{x} / V$.

The canonical transformation from $\left(p_{x}, x, p_{y}, y\right) \rightarrow$ $\left(J_{x}, \theta_{x}, J_{y}, \theta_{y}\right)$ is

$$
\begin{aligned}
& x=\frac{\pi}{2}+\sin ^{-1}\left[\kappa_{x} \operatorname{sn}\left(f_{x}, \kappa_{x}\right)\right], p_{x}=\sqrt{V} \kappa_{x} \operatorname{cn}\left(f_{x}, \kappa_{x}\right), \\
& y=\frac{\pi}{2}+\sin ^{-1}\left[\kappa_{y} \operatorname{sn}\left(f_{y}, \kappa_{y}\right)\right], p_{y}=\sqrt{V} \kappa_{y} \operatorname{cn}\left(f_{y}, \kappa_{y}\right),
\end{aligned}
$$

where $f_{x}=\frac{2}{\pi} \mathrm{K}\left(\kappa_{x}\right) \theta_{x}$ and $f_{y}=\frac{2}{\pi} \mathrm{K}\left(\kappa_{y}\right) \theta_{y}$. The Jacobian of this transformation is equal to one.

For the case $V=U / 2=10$, the lowest and highest values of energy are $E_{X}=0$ and $E_{x}=10$. Also, the lowest and highest values of the action variable are $J_{z}=0$ and $J_{x}=$ 2.013 , respectively.

\section{Action-angle variables: Rotation}

For energies $E_{x}>V$, the pendulum rotates and the action variable is defined

$$
J_{x}=\frac{2 \sqrt{E_{x}}}{\pi} \mathrm{E}\left(\kappa_{x}\right),
$$

where the modulus $\kappa_{x}^{2}=V / E_{x}$.

The canonical transformation from $\left(p_{x}, x, p_{y}, y\right) \rightarrow$ $\left(J_{x}, \theta_{x}, J_{y}, \theta_{y}\right)$ is

$$
\begin{aligned}
x & =\frac{\pi}{2}+\sin ^{-1}\left[\operatorname{sn}\left(g_{x}, \kappa_{x}\right)\right]=\frac{\pi}{2}+\operatorname{am}\left(g_{x}, \kappa_{x}\right), \\
p_{x} & =\frac{\sqrt{V}}{\kappa_{x}} \operatorname{dn}\left(g_{x}, \kappa_{x}\right), \\
y & =\frac{\pi}{2}+\sin ^{-1}\left[\operatorname{sn}\left(g_{y}, \kappa_{y}\right)\right]=\frac{\pi}{2}+\operatorname{am}\left(g_{y}, \kappa_{y}\right), \\
p_{y} & =\frac{\sqrt{V}}{\kappa_{y}} \operatorname{dn}\left(g_{y}, \kappa_{y}\right),
\end{aligned}
$$

where $g_{x}=\frac{2}{\pi} \mathrm{K}\left(\kappa_{x}\right) \theta_{x}$ and $g_{y}=\frac{2}{\pi} \mathrm{K}\left(\kappa_{y}\right) \theta_{y}$ and $\mathrm{K}(\kappa)$ is the complete elliptic integral of the first kind. The Jacobian of this transformation is equal to one.

For the case $V=U / 2=10$, the lowest and highest values of energy are $E_{x}=10$ and $E_{x}=\infty$. Also, the lowest and highest values of the action variable are $J_{z}=2.013$ and $J_{x}=$ $\infty$, respectively.
[1] V. I. Arnold, Sov. Math. Dokl. 5581 (1964); (reprinted in R. S. MacKay and J. D. Meiss, Hamiltonian Dynamical Systems (Adam Higler, Bristol, 1987).

[2] A. N. Kolmogorov, Dokl. Akad. Nauk SSSR 98, 527 (1954) [English translation in R. Abraham, Foundations of Mechanics (W.A. Benjamin, New York, 1967), Appendix D].

[3] V. I. Arnold, Russ. Math. Surv. 18, 9 (1963).

[4] J. Moser, Comm. Pure Appl. Math. 11, 81 (1958).

[5] N. N. Nekhoroshev, Funct. Anal. Appl. 5, 338 (1971).

[6] N. N. Nekhoroshev, Russ. Math. Surv. 32, 1 (1977).

[7] B. Chirikov, Phys. Rep. 52, 263 (1979).

[8] C. Froeschle, M. Guzzo, and E. Lega, Science 289, 2108 (2000).

[9] E. Lega, M. Guzzo, and C. Froeschle, Physica D 182, 179 (2003).

[10] A. J. Lichtenberg and M. A. Lieberman, Regular and Chaoitic Dynamics, 2nd ed. (Springer-Verlag, New York, 1991).

[11] L. E. Reichl, The Transition to Chaos, 2nd ed. (Springer-Verlag, New York, 2004).

[12] S. Wiggins, Global Bifurcations and Chaos (Springer-Verlag, New York, 1988).

[13] P. M. Cincotta, New Astron. Rev. 46, 13 (2002).

[14] J. von Milczewski, G. H. F. Diercksen, and T. Uzer, Phys. Rev. Lett. 76, 2890 (1996).

[15] K. Kaneco and R. J. Bagley, Phys. Lett. A 110, 435 (1985).
[16] A. I. Malyshev and L. A. Chizhova, J. Exp. Theor. Phys. 110, 837 (2010).

[17] K. Nakamura and T. Harayama, Quantum Chaos and Quantum Dots (Oxford University Press, Oxford, 2004).

[18] L. E. Reichl, Phys. Rev. A 39, 4817 (1989).

[19] G. O. Morrow and L. E. Reichl, Phys. Rev. A 50, 2027 (1994).

[20] A. Hemmerich, D. Schropp, and T. W. Hansch, Phys. Rev. A 44, 1910 (1991).

[21] M. Greiner, I. Bloch, O. Mandel, T. W. Hansch, and T. Esslinger, Appl. Phys. B 73, 769 (2001).

[22] I. Bloch, J. Dalibard, and Sylvain Nascimbene, Nat. Phys. 8, 267 (2012).

[23] M. Greiner, O. Mandel, T. Esslinger, T. W. Hansch, and I. Bloch, Nature (London) 415, 39 (2002).

[24] E. Horsley, S. Koppell, and L. E. Reichl, Phys. Rev. E 89, 012917 (2014).

[25] D. A. Steck, W. H. Oskay, and M. G. Raizen, Science 293, 274 (2001).

[26] D. A. Steck, W. H. Oskay and M. G. Raizen, Phys. Rev. Lett. 88, 120406 (2002).

[27] R. Luter and L. E. Reichl, Phys. Rev. A 66, 053615 (2002).

[28] W. K. Hensinger, H. Haffner, A. Browaeys, N. R. Heckenberg, K. Helmerson, C. McKenzie, G. J. Milburn, W. D. Phillips, and 
S. L. Rolston, H. Rubinsztein-Dunlop, and B. Upcroft, Nature (London) 412, 52 (2001).

[29] H. Hsu and L. E. Reichl, Phys. Rev. B 74, 115406 (2006).

[30] T.-R. T. Han, F. Zhou, C. D. Malliakas, P. M. Duxbury, S. D. Mahanti, M. G. Kanatzidis, and C.-Y. Ruan, Sci. Adv. 1, e1400173 (2015).
[31] P. F. Byrd and D. Friedman, Handbook of Elliptic Integrals for Engineers and Scientists (Springer-Verlag, Berlin, 1971).

[32] R. Graham, M. Schlautmann, and P. Zoller, Phys. Rev. A 45, R19 (1992).

[33] B. P. Holder and L. E. Reichl, Phys. Rev. A 76, 013420 (2007). 